\title{
The sequential view: From rapidly fading stimulus traces to the organization of memory and the abstract concept of number
}

\author{
E. J. CAPALDI \\ Purdue University, West Lafayette, Indiana
}

\begin{abstract}
The development of the sequential approach to instrumental learning from about 1958 to the present is described. The sequential model began as an attempt to explain a particular class of neglected partial reward phenomena, those in which performance in acquisition and extinction is influenced by the particular sequence in which rewarded and nonrewarded trials occur in acquisition, and it was subsequently applied to a variety of other phenomena. Over time, the sequential model grew, sometimes through the replacement of older assumptions by novel ones, as when retrieved memories replaced stimulus traces, and sometimes simply through the addition of novel assumptions, such as that animals are capable of remembering retrospectively one, two, three or more prior nonrewarded outcomes-the $\mathrm{N}$-length assumption. The most recent assumption added to the sequential model is that on a given trial the animal may utilize its memory of prior reward outcomes to anticipate both the current reward outcome and one or more subsequent reward outcomes. One way to view the sequential model is to say that it is a specific theory in various degrees of competition with other specific theories. Several examples of this are provided. Another way to view the sequential model, a more important way in my opinion, is to see it as a representative of a general theoretical approach, intertrial theory, which differs in fundamental respects from another much more generally utilized theoretical approach, intratrial theory. I suggest that there is a substantial body of data that can be explained by intertrial mechanisms but not by intratrial mechanisms. The future may well reveal that the intertrial mechanisms have greater explanatory potential than the currently more popular intratrial mechanisms.
\end{abstract}

The cognitive abilities of rats are far greater and more varied than was dreamed of even remotely until recently. Even now they are familiar to only a relative few. As Wright (1992) has recently indicated, an animal may perform poorly not because of its cognitive limitations, but because the experimenter has employed a task ill suited to the particular species. Varied reward situations, emphasized in this paper, have demonstrated themselves to be ideally suited to revealing a variety of complex abilities in rats. To be sure, varied reward situations were employed initially to test the simplest of hypotheses. But varied reward situations, as we shall see, are very flexible, and when appropriately modified they can be employed in the service of many ends. Within the context of the author's sequential hypothesis, varied reward tasks have been repeatedly modified to reveal increasingly complex processes in the rat, an activity that is ongoing.

In the present paper, varied reward refers to situations with the following characteristics. Animals receive two or more different reward outcomes, such as food reward

Correspondence concerning this article should be sent to E. J. Capaldi, Department of Psychological Sciences, Purdue University, 1364 Psychology Building, West Lafayette, IN 47907-1364 (e-mail: julie@brazil. psych.purdue.edu). and nonreward either intermixed in the same phase or successively in separate phases. The differential reward outcomes are not validly signaled by differential exteroceptive cues. The sequential hypothesis began as an attempt to explain the effects of various sequences of reward and nonreward trials-a variety of partial reinforcement-on learning and performance. Over time, the sequential hypothesis has been applied to a variety of additional varied reward situations. These include the successive acquisition and extinction of responses, varied magnitude of reward, varied delay of reward, serial learning with reward events as stimulus items, counting reward events, organizing reward events into new functional units or chunks, and even conventional discrimination learning situations in which distinctive exteroceptive cues validly signal better and worse reward outcomes.

It is well to clarify at the outset my view of the relationship between the sequential hypothesis and instrumental varied reward situations. My view is that in some instances varied reward situations provide better insights into the processes that regulate learning and performance than do some other types of learning situations. In other instances, other situations may be as good or better. As a specific example, many years ago Capaldi and Spivey (1964), employing a varied reward procedure, provided 
evidence which suggested that rats were able to remember the specific reward outcome-reward or nonrewardthat had occurred $24 \mathrm{~h}$ previously. More recently, Overman and Doty (1980), employing a delayed matching to sample procedure, provided evidence that monkeys were able to remember a visual stimulus over a 24 -h retention interval. In this instance, a varied reward task and a delayed matching to sample task were about equally effective in indicating that animals were able to remember specific episodic events over long retention intervals. I have no doubt that for some purposes the delayed matching to sample situation is superior to varied reward tasks. However, in my view, the opposite is also the case. To be specific, I cannot easily imagine that the complex organizational capacities clearly exhibited by rats in certain varied reward tasks could be revealed nearly as well in the delayed matching to sample task or in a variety of other learning situations - such as Pavlovian conditioning.

Varied reward tasks taken collectively, and partial reward in particular, have had and continue to have a major impact on contemporary theory. As early as 1939, a spate of partial reinforcement investigations had been reported which clearly signaled troubled waters for extant theoretical approaches. However, as perhaps often occurs when new perplexing data are reported, it was not immediately recognized how seriously the novel findings were to be taken. Hull, for one, may not have made up his mind about this. On the one hand, in 1941 in his seminar at Yale, he described a mechanism, later to be called the Hull-Sheffield hypothesis, that was capable of explaining the limited available partial reward data (see Jenkins \& Stanley, 1950). On the other hand, 2 years later, in his very influential Principles of Behavior (1943), Hull barely mentioned partial reinforcement, saying only that he realized it had the potential to create problems for his theory (p. 337). In any event, Hull's 1952 book presented a marked contrast to his 1943 statement in that it devoted considerable space to an attempt to explain a variety of varied reward effects.

By 1950 , it had become unmistakably clear that new theories had to be devised in order to explain why partial reward produced greater resistance to extinction than consistent reward did-the partial reinforcement extinction effect. Does our concern with explanations of the partial reinforcement extinction effect and related phenomena have the same significance today as it did in 1940 or 1950 ? I think it does. Consider the following: Data reported recently (e.g., Capaldi \& Miller, 1988a, 1988b) suggest that rats are able to count reward events and to categorize the number of reward events in an abstract manner. Other data reported recently suggest that rats are able to remember a number of reward events by organizing them into a new functional unit or chunk, called a list chunk, which can be employed as a discriminative cue for another series of reward events that might occur as much as $20 \mathrm{~min}$ later (Capaldi, Miller, Alptekin, \& Barry, 1990). According to the sequential approach, the partial reinforcement extinction effect, the ability of rats to count and to ab- stractly categorize reward events, the ability of rats to organize reward events in a complex manner over long retention intervals, and numerous other phenomena are related in that all are united by a common set of principles.

As might be gathered from the preceding comments, theories capable of dealing with varied reward data have evolved over decades, and they are still evolving as novel, unexpected varied reward phenomena continue to be reported. In connection with varied reward data, three things should be kept in mind. No available theory is capable of explaining all of the very considerable body of varied reward data. Newer varied reward data suggest that even the simple rat is a creature of enormous cognitive complexity - that rats possess far greater organization and anticipatory abilities than has generally been realized. Finally, despite our collective complete lack of success in explaining all varied reward data, at least some of the more salient explanatory principles seem to have been discovered.

In my view, the three most basic and fundamental principles isolated to date for understanding varied reward effects are the following. First, different interoceptive, reward-produced stimuli are associated with different conditions of varied reward, and these interoceptive stimuli control instrumental behavior strongly, more strongly in many instances than any differential exteroceptive stimuli an experimenter may or may not explicitly manipulate. More simply put, animals trained under different conditions of varied reward learn different things, sometimes vastly different things, and thus perform differently. This assumption is, of course, incorporated into the author's sequential approach just as it is in some other approaches. However, within the sequential approach, the emphasis on the variety of complex reward events represented by animals is considerably greater than it is in other approaches. Second, the memories of two or more different reward events may be fused, as it were, into a new functional unit or chunk that exercises control over behavior. Third, among the properties of the chunk is that it can function as a rule or a concept. To put the assumptions of the sequential approach in better focus, this paper is divided into three sections. The first emphasizes some of the circumstances involved in the original development of the sequential approach, with its emphasis on memory of individual reward events. The second deals with newer experimental findings that have led to more recent ideasto the emphasis on organizing individual reward events into larger functional units. In the third section, which is speculative, directions are considered for further development of the sequential approach, with an emphasis on anticipation of reward events to go along with memory of reward events.

\section{THE SEQUENTIAL VIEW: INITIAL DEVELOPMENT}

In his review of the literature, Lewis (1960) expressed dissatisfaction with available explanations of the partial 
reinforcement extinction effect and a variety of related phenomena. Lewis did describe Amsel's promising and subsequently influential frustration theory, which had appeared only 2 years earlier in the Psychological Bulletin (Amsel, 1958). While Amsel's view was reasonably well developed by 1960 , the sequential view was not, although Lewis did cite two papers containing some of the seeds of the sequential view (Capaldi, 1957, 1958). While a number of hypotheses have been applied to this or that varied reward situation, only three have been applied to a sufficiently broad array of such data to be considered general: Amsel's frustration hypothesis (e.g., Amsel, 1992); the author's sequential hypothesis; and the Daly and Daly (1982) view, which utilizes principles from Amsel's theory and that of Rescorla and Wagner (1972).

The difficulty posed for various theories by the partial reinforcement extinction effect and a variety of other varied reward effects may be illustrated with reference to Hull's 1943 theory. According to Hull's approach in his 1943 book, animals receiving different schedules of reward formed the same associations but at different strengths. Habits (stimulus-response, or S-R, bonds) were strengthened on rewarded trials; the bigger and the more immediate the reward, the greater the asymptote of habit growth. Call this approach strength theory. According to Hull, as habit strength increased, so too should resistance to extinction. But where number of trials is equated, habit strength cannot be greater under partial reward than under consistent reward, because, of course, under partial reward not all trials are rewarded. Clearly a reliance on strength theory, in the absence of additional assumptions, will not do as an explanation of the partial reinforcement extinction effect and of varied reward data generally.

The frustration hypothesis and the sequential hypothesis share two major features which allow each hypothesis to deal adequately with a wide range of varied reward effects. Both views assume that extinction involves generalization decrement. That is, over extinction trials, animals progressively encounter novel stimuli unlike those which control responding in acquisition, and so they progressively manifest response decrement. In addition, and much more importantly, both theories assume that as schedule of reward varies, learning may be affected not only quantitatively (strength theory) but also qualitatively. More specifically, both theories assume that distinctive reward events produce distinctive stimuli that acquire control over responding. The latter assumption, it may be strongly argued, is indispensable to an understanding of the wide array of available varied reward effects and thus instrumental learning in general. Yet this assumption has been ignored in many recent theories. As merely one example, the Rescorla-Wagner (1972) model, the most influential model of recent times, is a strength theory which assumes that extinction involves unlearning. It is true that the Rescorla-Wagner model was devised initially to explain Pavlovian phenomena, but many have applied it to instrumental learning as well. In a few of these applications, it has been recognized that the RescorlaWagner model is incapable of explaining instrumental varied reward data in the absence of postulating rewardproduced cues (e.g., Daly \& Daly 1982; Haggbloom \& Capaldi, 1992; Neely \& Wagner, 1974).

Reward-produced cues possess a variety of attributes. The emotional attributes of reward reduction have been much more heavily emphasized by the frustration model than by the sequential approach. The emotional emphasis has enabled the frustration view to explain phenomena that the sequential view cannot. For example, the frustration view can explain why partial reinforcement produces faster running than consistent reinforcement does in the start section of a runway, but slower running in the goal section-as well as various other related phenomena (see, e.g., Amsel, 1967). The sequential hypothesis, however, was devised to explain a rather different class of varied reward phenomena-those clearly sensitive to the particular sequence or order in which different reward events are presented. Few hypotheses have had much to say about such clearly sequential phenomena, even today, and those which have have dealt with only a limited range of them, most notably single alternation pattern learning (see, e.g., Amsel, 1992; Daly, 1991). In any case, as data were collected it became clear that the distinction between clearly sequential phenomena and other phenomena was not tenable: performance under all reward schedules, even irregular schedules of rewarded and nonrewarded trials, seemed to be regulated by sequential variables. But this was to become clear later.

By 1960 , only a few clearly sequential effects had been reported (see Lewis, 1960), but one reported by Tyler, Wortz, and Bitterman (1953), pattern running, had a profound effect on the author. Tyler et al. provided rats in a runway with a single alternating series of rewarded and nonrewarded trials at a relatively short $(30-\mathrm{sec})$ intertrial interval (ITI). Quite interestingly, with training, the rats came to run faster on rewarded than on nonrewarded trials. Such single alternation pattern running is, of course, an unconventional form of discrimination learning. In conventional discrimination learning problems, as indicated, differential exteroceptive cues signal rewarded trials ( $\mathrm{S}+$ cue) and nonrewarded trials ( $\mathrm{S}-$ cue). In single alternation pattern running, there are no exteroceptive $S+$ and $S-$ cues. In 1960, only one theory capable of explaining single alternation pattern learning was available, the HullSheffield hypothesis, and that theory was known by then to have a variety of serious problems.

According to the Hull-Sheffield hypothesis (see Sheffield, 1949), partial reward, unlike consistent reward, could allow extinction-like stimuli to be conditioned to the instrumental response in acquisition. This would result in a partial reinforcement extinction effect, because in extinction, the extinction-like stimuli would elicit the reaction more strongly in the partial group than in the consistent group, which would experience considerable generalization decrement. For all the many differences between the Hull-Sheffield hypothesis, the frustration 
hypothesis, and the sequential hypothesis, an emphasis on a generalization decrement explanation of the partial reinforcement extinction effect is common to all. In any event, the stimuli postulated by the Hull-Sheffield hypothesis were assumed to be rapidly fading stimulus traces. Thus, following rewarded trials, the traces might consist of food particles in the mouth. Following nonrewarded trials, the traces might be those of frustration. All stimuli, including stimulus traces, were assumed to be conditioned to the instrumental reaction on rewarded trials. In acquisition, therefore, traces of frustration could be conditioned to the reaction on the rewarded trials that followed nonrewarded trials at a short ITI. Thus, transitions from nonrewarded to rewarded trials, or NR transitions, were given central theoretical status by the Hull-Sheffield hypothesis-a major development, as it turned out. In any event, the Hull-Sheffield hypothesis could explain single alternation pattern learning as follows. Traces of rewarded events, by occurring on nonrewarded trials, would become inhibitory (run slow); traces of nonrewarded events, by occurring on rewarded trials, would become excitatory (run fast). Unfortunately for the Hull-Sheffield hypothesis, Tyler et al. (1953) reported that a 50\% irregular schedule of partial reward produced greater resistance to extinction than a single alternation schedule did, despite the fact that the single alternation schedule contained the greater number of NR transitions.

While still other findings created problems for the HullSheffield hypothesis (see, e.g., Lewis, 1960), the single greatest problem was undoubtedly this: many investigators reported obtaining a partial reinforcement extinction effect at long ITIs (see, e.g., Lewis, 1960; Wilson, Weiss, \& Amsel, 1955). According to the Hull-Sheffield hypothesis, of course, a partial reinforcement extinction effect should not be obtained at long ITIs, because stimulus traces, the basis of the partial reinforcement extinction effect, fade rapidly. If anything, according to the HullSheffield hypothesis, at long ITIs partially rewarded animals should extinguish more rapidly than consistently reinforced animals. By 1960 , then, some phenomena were available which could be taken to suggest that in some respects the Hull-Sheffield hypothesis was on the right track (single alternation pattern learning), while other phenomena suggested that the hypothesis was in other respects unworkable (the partial reinforcement extinction effect at long ITIs). Most theorists emphasized the unworkability of the sort of mechanisms emphasized by the HullSheffield hypothesis (see, e.g., Amsel, 1962; Lewis, 1960). As another example, commenting on the shift in theorizing about the partial reinforcement extinction effect that had taken place from, say, 1940 to 1960 , Lawrence and Festinger (1962) said, "The major shifts in these accounts over the years has been from an emphasis on intertrial sources of stimulation, as in the Sheffield account, to a reliance on intratrial factors such as frustration responses and competing behaviors" (p. 29). I shall consider the issue of intertrial versus intratrial theories later, after a variety of relevant data have been reported. In this connection, one may view the sequential view, as I have so far, as a specific theoretical position in competition with other specific theoretical positions. Later I shall view the sequential view as a representative of an intertrial approach to learning, one in competition with the far more numerous and conventional intratrial approaches. Intratrial approaches limit analysis to events occurring within a trial (see, e.g., Amsel, 1962, 1992; Lawrence \& Festinger, 1962). In any case, to the writer at least, single alternation pattern learning seemed to indicate that intertrial sources of stimulation were important at least under some conditions. But what conditions? It should be noted that Amsel (1992) and Daly (1991), in applying frustration theory to a variety of developmental data, have postulated an intertrial mechanism to explain single alternation pattern learning in infant rats. Thus, the frustration view employs, in addition to its intratrial mechanisms, some version of an intertrial theory, albeit a vastly simplified form relative to the sequential approach. As will become clear later in this paper, the sequential model has come to accept certain assumptions of frustration theory.

\section{Representations of Reward Events at Long ITIs}

Do interoceptive stimuli associated with rewarded and nonrewarded trials really fade rapidly as Hull and Sheffield suggested? Currently available evidence strongly indicates that such stimuli may be functional (or nonfunctional) at any ITI, long or short. Capaldi and Stanley (1963) reported that rats given a single alternation schedule at a 20-min ITI manifested pattern running that compared favorably to that of rats trained at various shorter ITIs. Capaldi and Spivey (1964), in a previously mentioned investigation, subsequently reported single alternation pattern running at a $24-\mathrm{h}$ ITI. Some failed to replicate our findings obtained at long ITIs (e.g., Gonzalez $\&$ Bitterman, 1969). Some years later, single alternation pattern learning (and other sequential effects) was obtained by Mellgren and coworkers in the Oklahoma laboratory at a 24-h ITI, and the reason for the discrepancy in prior results was isolated (Jobe, Mellgren, Feinberg, Littlejohn, \& Rigby, 1977; see also Jobe \& Mellgren, 1974). In brief, the better the conditions for memory retrieval (see below), the better the single alternation pattern running, and the better could other sequential effects be obtained. By about 1963 , the sequential view was similar in some respects to the Hull-Sheffield hypothesis. One major difference was this: I rejected the idea that in order to explain varied reward effects two types of theory had to be employed-an intertrial model for short ITIs, and an intratrial model for long ITIs (see, e.g., Amsel, 1967). Given the single alternation pattern learning findings at long ITIs, we reasoned that an intertrial model could be applied to long as well as short ITIs.

\section{Memory Retrieval}

In addition to being functional at long retention intervals, do the interoceptive stimuli produced by reward 
events have other memory-like characteristics? That they do was demonstrated in a series of studies employing intertrial reinforcement. In the first of these investigations, Capaldi, Hart, and Stanley (1963) provided rats with two sorts of trials-conventional runway trials and direct placement into a baited goalbox. These placements were called intertrial reinforcements. They found that if the intertrial reinforcements were administered in the ITI separating nonrewarded trials from rewarded trials, extinction was rapid relative to a variety of controls. Why do intertrial reinforcements given in the ITI between nonrewarded and rewarded trials reduce resistance to extinction? We reasoned as follows. It may be assumed that on the nonrewarded trial, the memory of nonreward, $S^{N}$, was stored. On subsequent intertrial reinforcement trials, two noteworthy events would occur: (1) $\mathrm{S}^{\mathrm{N}}$ would be retrieved but would not become a signal for responding, since no response occurred; and (2) the memory of reward, $S^{R}$, would be stored. On the rewarded trial following the intertrial reinforcement trial, $S^{R}$, the last stored memory, would be retrieved. Thus, according to our reasoning, when intertrial reinforcement was given in the ITI separating nonrewarded from rewarded trials, it prevented $S^{N}$ from being conditioned to the instrumental reaction, converting a schedule of partial reward into a (functional) schedule of consistent reward. Thus, extinction could be rapid following partial reward.

Quite notable among our intertrial reinforcement findings were those reported by Capaldi and Spivey (1963). They reported that if the intertrial reinforcements occurred in a goalbox of a different brightness than that of the runway goalbox, substantial resistance to extinction was obtained; that is, intertrial reinforcement failed to reduce the partial reinforcement extinction effect. This finding can be interpreted to mean that $S^{N}$ stored in a given context (e.g., black runway goalbox) would not be strongly retrieved in a different context (e.g., white intertrial reinforcement goalbox) but would be retrieved when the original context, the black runway goalbox, was presented. Thus when the runway and intertrial reinforcement goalboxes differed, it was assumed that $S^{N}$ was nevertheless conditioned to the instrumental reaction, thereby increasing resistance to extinction. This interpretation was supported by findings indicating that various sequential phenomena such as single alternation pattern running at a $24-\mathrm{h}$ ITI were more easily obtained when runway and goalbox were similar rather than different (Jobe \& Mellgren, 1974; Jobe et al., 1977). When Gonzalez and Bitterman (1969) failed to obtain single alternation pattern running at long ITIs, the startbox and goalbox differed.

Other conditions under which intertrial reinforcements were or were not effective in reducing resistance to extinction were reported, generating in several instances controversies eventually resolved in accordance with sequential principles (see, e.g., Black \& Spence, 1965; Capaldi, Miller, Alptekin, Barry, \& Haggbloom, 1991; Capaldi \& Olivier, 1967; Capaldi \& Wilson, 1968; Rudy, Homzie, Cox, Graeber, \& Carter, 1970; Spence, Platt, \& Matsumoto, 1965).
Following Capaldi and Spivey's (1963) finding that the memories $S^{R}$ and $S^{N}$, once stored, would tend to be retrieved to the extent that the storage and retrieval contexts were similar, we asked, are rats aware of how much time has elapsed between trials? And, if they are thus aware, does this temporal representation function as a retrieval cue? That is, we hypothesized that the time elapsing between trials is a stimulus utilized by rats. As an example, assume that a series of trials occurs at a 30 -sec ITI and that on the last trial of this series, a nonrewarded trial, $\mathbf{S}^{\mathbf{N}}$, is stored. If, in the same apparatus, the next trial of another series, the retention interval, should occur $30 \mathrm{sec}$ later, we could be sure on the basis of then available data that $S^{\mathbf{N}}$ would be retrieved. But would $\mathbf{S}^{\mathbf{N}}$ be retrieved if the next trial occurred, for example, $20 \mathrm{~min}$ later? We thought that $S^{N}$ would not be retrieved so well under those conditions-not because the retention interval was $20 \mathrm{~min}$, but because the retention interval of $20 \mathrm{~min}$ differed from the storage interval of $30 \mathrm{sec}$. Thus we also thought that $S^{N}$ would be poorly retrieved if the opposite conditions prevailed-that is, if the storage interval was, for example, $20 \mathrm{~min}$, with the retention interval being $30 \mathrm{sec}$. Our hypothesis, then, was that a memory stored at a given ITI could be strongly retrieved at that ITI, but would be poorly retrieved at some other ITI, either longer or shorter.

Our hypothesis concerning the role of ITI in memory retrieval was confirmed initially when we employed the successive acquisition and extinction of responses, and subsequently by a variety of other phenomena. Consider, first, how the sequential view would be applied to the successive acquisition and extinction of reactions. In that situation, acquisition and extinction sessions alternate. According to the sequential hypothesis, in the initial acquisition session under consistent reinforcement, the memory of reward, $S^{R}$, becomes a signal for reward and forms an excitatory association with the instrumental reaction. In extinction, the memory of nonreward, $S^{\mathrm{N}}$, becomes a signal for nonreward and forms an inhibitory association with the instrumental reaction. Extinction does little to reduce the capacity of $S^{R}$ to signal reward and elicit responding; thus acquisition rate should increase over sessions, as has been found in such studies. Moreover, reacquisition does little either to decrease the capacity of $\mathrm{S}^{\mathrm{N}}$ to signal nonreward, or to inhibit responding, and so extinction rate should increase over sessions. Now, if for some reason in a successive acquisition and extinction study a rewarded response occurred in the presence of $S^{N}$, this would increase the capacity of $S^{N}$ to signal reward and thus to elicit the instrumental response. This would not allow the extinction rate to increase over sessions, or would at least slow the rate of increase. This could occur in one way if nonrewarded memories associated with extinction sessions were retrieved on reacquisition sessions. I will consider this below.

In the successive acquisition and extinction situation, extinction rates were said to vary with ITI, a hypothesis consistent with then available data; this circumstance was considered favorable for competing response theory (see, 
e.g., Lauer \& Estes, 1955). For example, under massed trial conditions, extinction rate was found to increase over sessions (Bullock \& Smith, 1953; Perkins \& Cacioppo, 1950), whereas under spaced trial conditions, extinction rates did not increase over sessions and could decrease (Lauer \& Carterette, 1957; Lauer \& Estes, 1955). Capaldi, Leonard, and Ksir (1968) showed that ITI per se was not the critical factor, and that either rate of extinction could be obtained, depending on whether the ITI separating the extinction session from the reacquisition session was the same as or different from the ITI separating other trials in extinction and reacquisition. They reported that if the two ITIs were the same, the extinction rate would not increase over sessions, but that if the two ITIs were different, the extinction rate would increase over sessions. It so happened that in the previous spaced trial studies, the two ITIs were similar, whereas in the previous massed trial studies, the two ITIs tended to differ. For example, in the so-called massed trial studies, the extinction trials would be massed and the reacquisition session would occur $24 \mathrm{~h}$ later. Under these conditions, $\mathbf{S}^{\mathbf{N}}$ stored at a short ITI would not be retrieved well at the long ITI, so it would not acquire a substantial capacity to elicit responding; thus, extinction rate would tend to increase over sessions. In the previous spaced trial studies, the extinction ITI and the reacquisition ITI tended to be alike, which would allow $S^{N}$ to acquire control over responding, thereby producing a decreased rate of extinction over sessions. Perhaps the most surprising result reported by Capaldi et al. (1968), given the then prevailing view that memory retrieval is impaired by the passage of time, was that $S^{N}$ stored at a long ITI was more strongly retrieved at a long ITI than at a short ITI. To my knowledge, no other hypothesis has attempted to explain the full range of successive acquisition and extinction findings that have been reported (see also Leonard \& Capaldi, 1971). The idea that time between trials may function as a retrieval cue has been confirmed subsequently in a variety of preparations: pattern running under various reward schedules, partial reinforcement extinction, and a conventional discrimination task (see, e.g., Capaldi et al., 1990; Capaldi, Nawrocki, Miller, \& Verry, 1986b).

The reacquisition of extinguished responses has recently come under examination in the Pavlovian situation, with results similar to those obtained in the instrumental situation when extinction and reacquisition ITIs differ (see, e.g., Bouton, 1991). Interestingly, Bouton (1991) has suggested that the reacquisition effect, as well as some other Pavlovian effects, may involve stimuli associated with the unconditioned stimulus or US (see also Bouton, 1993; Bouton, Rosengard, Achenbach, Peck, \& Brooks, 1993). According to Bouton et al., "USs themselves have stimulus properties that may gain control over conditioned responding', (p. 90). Of course, US events in the Pavlovian situation correspond to reward events in the instrumental situation. Possibly, then, successive acquisition and extinction effects, and perhaps other phenomena, are due to similar mechanisms of stimulus control in Pavlovian and instrumental tasks.

\section{Memory for Multiple Reward Events}

Single alternation pattern running, among other findings, demonstrated that rats are capable of remembering the prior reward outcome. Are rats capable of remembering two or more prior reward outcomes? Rats are, in fact, able to do this. Let us refer to the number of nonrewarded trials that precede a rewarded trial as $\mathrm{N}$-length. For example, if one nonrewarded trial precedes a rewarded trial (NR), $\mathrm{N}$-length is one. If two nonrewarded trials in succession precede a rewarded trial (NNR), Nlength is two, and so on. In a single alternation schedule, $\mathrm{N}$-length is one. In a $50 \%$ irregular schedule, $\mathrm{N}$-lengths of one, two, and three might occur (see, e.g., Tyler et al., 1953). I entertained the assumption that rats are capable of remembering, within limits, how many nonrewarded trials precede a rewarded trial-one $\left(\mathrm{S}^{\mathbf{N} 1}\right)$, two $\left(\mathrm{S}^{\mathrm{N} 2}\right)$, three $\left(S^{\mathrm{N} 3}\right)$, and so on-with the memory associated with a particular $\mathrm{N}$-length becoming a signal for reward (Capaldi, 1964). Extinction consists of long N-lengths. It is intuitively clear that, other things being equal, a stimulus occurring late in extinction-say, $S^{\mathrm{N} 15}$-would receive greater generalized excitatory capacity to elicit responding from the more similar $S^{N 3}$ than from the less similar $S^{N 1}$. Note that the N-length assumption can be used to explain cases in which the percentage of reward is held constant, with the sequence of nonrewarded and rewarded trials being varied (e.g., single alternation vs. $50 \%$ irregular reward), and also cases in which the percentage of reward varies. This is because, generally speaking, lower percentages of reward involve longer $\mathrm{N}$-lengths, which may occur more frequently, unless, of course, steps are taken to prevent this, something we have done in some investigations. In any event, Bacon (1962) varied the percentage of reward $(30 \%, 50 \%, 70 \%$, and $100 \%)$ and number of acquisition trials $(10,30,100$, and 300$)$, obtaining extinction findings totally in agreement with an $\mathrm{N}$-length analysis; that is, resistance to extinction increased with lower percentages of reward and increased training.

When one is evaluating $\mathrm{N}$-length, matters become complicated, unfortunately, because the effects of training level cannot be ignored. For example, when the number of acquisition trials is equated under a single alternation schedule, $S^{\mathrm{N} 1}$ may occur more frequently than $S^{\mathrm{N} 1}, S^{\mathrm{N} 2}$, and $S^{\mathrm{N} 3}$ put together in a $50 \%$ irregular schedule. Consider the effects of this. Under conditions of limited acquisition training, the excitatory capacity acquired by $\mathbf{S}^{\mathrm{N} 1}$ under the single alternation schedule will be much greater than that acquired by each of the memories $S^{\mathrm{N} 1}, S^{\mathrm{N} 2}$, and $\mathrm{S}^{\mathrm{N} 3}$ under the irregular schedule. Thus, following limited acquisition training, extinction stimuli such as $S^{\text {N15 }}$ might receive more generalized excitatory capacity from $S^{N 1}$ (single alternation schedule) than from $S^{N 1}, S^{N 2}$, and $\mathrm{S}^{\mathrm{N} 3}$ together (50\% irregular schedule). This would not be true following considerable acquisition training, since under both single alternation and irregular schedules $\mathrm{S}^{\mathrm{N} 1}$, $S^{\mathrm{N} 2}$, and $S^{\mathrm{N} 3}$ would approach the asymptote of excitatory strength. Consistent with this analysis is that, following limited acquisition training, the single alternation schedule produces greater resistance to extinction than the ir- 
regular schedule does, with the opposite being the case following more substantial acquisition training (see, e.g., Capaldi, 1958, 1964, 1967; Capaldi \& Hart, 1962). To mention some other extinction findings consistent with the $\mathrm{N}$-length analysis: higher percentages of reward schedules that contain longer $\mathrm{N}$-lengths than do lower percentages of reward schedules have produced the greater resistance to extinction; and the later in a series of nonrewarded trials that intertrial reinforcement is given, the faster extinction is (Capaldi, 1966; Capaldi \& Stanley, 1965; Capaldi \& Wilson, 1968). As N-length increases, resistance to extinction increases even at long ITIs (e.g., 30 min; Jobe \& Mellgren , 1974). In more recent times, the ability of rats to remember multiple reward events has been demonstrated not only by extinction data, but by acquisition data from serial leaning and counting tasks. These recent studies have shown that rats can remember multiple rewarded events, mixtures of rewarded and nonrewarded events, and multiple nonrewarded events (see e.g., Yazawa \& Fujita, 1984). The serial learning and counting studies will be mentioned in greater detail later.

\section{Other Varied Reward Effects}

There are a great number and variety of varied reward situations. To attempt to deal with this vast and diverse literature in depth would require much space and would not particularly serve to elucidate sequential principles further. In this section, only a few varied reward situations will be considered, and the emphasis will be on experiments in which the sequence of reward has been varied and which also have contributed in some way to a better understanding of the sequential approach. In this way, it should become possible in a reasonable space to introduce sequential principles that are applicable to a wide range of varied reward effects.

In one type of varied reward situation, the magnitude of reward is varied. Explaining the effects of magnitude of reward on learning and performance is a critical problem for any theory. To do this, the sequential model appeals to strength theory (see, e.g., Capaldi, 1967). It is a strength theory embedded in a memory framework, however, unlike the strength theories of Hull (1943) and Rescorla and Wagner (1972). It is assumed that as the magnitude of reward increases, the associative strength grows to higher asymptotes. Thus, if a nonrewarded trial is followed by large reward, an NL transition, $S^{\mathrm{N} 1}$ gains a greater capacity to elicit responding than if a nonrewarded trial is followed by small reward, an NS transition. Accordingly, NL transitions should supply greater amounts of generalized associative strength than NS transitions to memories of nonreward occurring in extinction and so should produce the greater resistance to extinction. This is precisely what has been found under both massed and spaced trial conditions (Capaldi \& Capaldi, 1970; Capaldi \& Lynch, 1968; Leonard, 1969). Sequential findings such as these, as a rule, can be generalized to investigations that are not explicitly sequential. For example, Wagner (1961), employing an irregular schedule of partial reward, reported that resistance to extinction was greater for large than for small reward. This finding can be explained in terms of frustration theory (see, e.g., Wagner, 1961). Of course, it can also be explained in sequential terms. However, frustration theory seems unable to explain why with overall magnitude of reward equated, NL transitions produce greater resistance to extinction than NS transitions (see e.g., Capaldi \& Capaldi, 1970; Leonard, 1969). Overall magnitude of reward was equated as follows: a group given the repeating sequence of three trials, SNL, was more resistant to extinction than a group given the three-trial sequence, LNS. Moreover, LNL produced greater resistance to extinction than did SNS, as in Wagner's investigation.

In partial delay of reward, some trials terminate in delayed reward and some trials terminate in immediate reward. In partial punishment situations, some trials terminate in a noxious stimulus-say, shock-and others in reward only. In varied magnitude of reward situations, some trials terminate in small reward, others in large reward. In each of these cases, resistance to extinction is affected by sequence of reward. That is, resistance to extinction is greater if transitions from worse reward (delayed reward, small reward, and shock) to better reward occur than if such transitions do not occur. The reason for this in sequential terms is by now perhaps obvious. It may be that each of the events (delayed reward, small reward, and shock) produce distinctive memories that are more similar to memories of nonreward than are memories of immediate reward or larger reward (see especially Capaldi, 1967; Capaldi \& Levy, 1972). The consequences of this are by now familiar.

I will briefly consider negative contrast effects. A group shifted to a series of small reward trials following a series of large reward trials may perform poorly, more poorly than a small reward control group-a successive negative contrast effect. The negative contrast effect has occurred after training with a large magnitude of partial reward, but not if the schedule of partial reward contains NL transitions (Capaldi \& Ziff, 1969). The sequential explanation for this is by now perhaps obvious. Conditioning of the memory of nonreward to the instrumental response will generalize to the memory of small reward. Of course, there are scores of contrast studies in which sequential variables have not been manipulated. The same is true, but less so, for partial delay, partial punishment, and varied magnitude of reward.

On the basis of the examples provided in this section, the approach of the sequential model to a variety of varied reward effects can be given in a few words. The sequential model asks: What reward-produced memory is conditioned to the instrumental response at what strength in acquisition and how similar is that memory to memories occurring in test?

\section{Discrimination Learning and Sequential Theory}

In a discrimination task, better reward outcomes, a higher percentage of reward, a larger reward magnitude, 
and so forth, are associated with one stimulus, the $\mathrm{S}+$ cue, than are associated with some other stimulus, the $S$ cue. Of course, a single alternation schedule, normally classified as a schedule of partial reinforcement, is a variety of discrimination learning, with $S^{N}$ being the $S+$ cue and $S^{R}$ being the $S-$ cue. Extinction also involves discrimination learning according to the sequential view. As an example, consider extinction following consistent reinforcement. According to the sequential view, $S^{R}$ would be the $S+$ cue in acquisition, with $S^{N}$ being the $S-$ cue in extinction.

In speaking of discrimination learning here, however, I refer to what is conventionally considered to be discrimination learning-the case in which some better reward outcome is associated with one exteroceptive cue (e.g., black) than with another exteroceptive cue (e.g., white). In the explicit discrimination learning case, the bulk of the sequential work has occurred in connection with brightness differential conditioning, the case in which a better reward outcome is associated with a response in, say, a black runway than in a white runway. Running speed is the dependent variable in that situation. Discriminative responding consists of faster running in $\mathrm{S}+$ than in $\mathbf{S}-$. Some sequential work has been carried out in the choice situation, particularly the T maze (see, e.g., Capaldi, Alptekin, Miller, \& Barry, 1992). Unless otherwise specified, however, I will be referring to brightness differential conditioning.

According to a variety of theories, the explanation of discrimination learning involves, as an indispensable first step, the identification of the stimuli that are controlling behavior. Such identification was a primary concern of early attention theories (see, e.g., Sutherland \& Mackintosh, 1971), just as it was in the controversy between continuity theory (e.g., Spence, 1936, 1937) and noncontinuity theory (e.g., Lashley, 1942). According to a variety of theories, including the three identified above, discriminative responding can be understood completely on the basis of the control exercised by the exteroceptive $\mathrm{S}+$ and $S-$ cues differentially reinforced by the experimenter, a view still commonly suggested (see, e.g., Williams, 1989). The view does not seem tenable: a substantial body of research reveals, for example, that the functional stimulus in discrimination situations consists of a compound of the $\mathrm{S}+$ and $\mathrm{S}-$ cues on the one hand, and the retrospective memories $\mathbf{S}^{\mathbf{R}}$ and $\mathbf{S}^{\mathbf{N}}$ on the other (see, e.g., Haggbloom \& Capaldi, 1992). In short, in discrimination learning, as in varied reward situations, the behavioral control exercised by the retrospective memories of reward and nonreward must be taken into account. This should hardly be surprising; as indicated, the line between varied reward situations and explicit discrimination learning situations is arbitrary.

In discrimination tasks, on transitions from one trial to the next within an alternative, either within $\mathrm{S}+$ or within $\mathrm{S}-$, memories of prior events are retrieved, as far as is currently known, in the same manner as they are in runways. Thus on a transition from, say, a rewarded trial in $\mathrm{S}+$ to another trial in $\mathrm{S}+$, the operative stimulus compound would be $S^{R}, S+$ and on a transition from a nonrewarded trial in $\mathrm{S}-$ to a trial in $\mathrm{S}-$, the operative stimulus compound would be $S^{\mathrm{N}}$, S-. On trial transitions between alternatives from $S-$ to $S+$ and from $S+$ to $S-$, the retrieval situation is a bit more complicated, as we shall see. For now it is enough to indicate that on a transition from a nonrewarded trial in $\mathbf{S}-$ to a subsequent trial in $\mathrm{S}+$ the operative stimulus compound may be $\mathrm{S}^{\mathrm{N}}, \mathrm{S}+$. And on a transition from a rewarded trial in $\mathrm{S}+$ to a subsequent trial in $\mathrm{S}-$, the operative stimulus compound may be $\mathrm{S}^{\mathrm{R}}, \mathrm{S}-$.

Behavior is a result of the capacity of each of the cues in the compound to elicit responding. For example, if late in training the animal is responding in the presence of a highly inhibitory $S-$ cue but a highly excitatory $S^{N}$ cue, responding in S- would be reasonably strong if not vigorous. Three comments are relevant here. First, the behavioral control acquired by $S^{R}$ and $S^{N}$ will depend to a considerable extent on the sequence of $\mathrm{S}+$ and $\mathrm{S}-$ trials. Second, many of the major effects obtained in discrimination learning situations depend on the excitatory capacity acquired by $\mathrm{S}^{\mathrm{N}}$, which of course acquires excitatory capacity by occurring on rewarded trials. Third, it is helpful to consider the early and late trials of discrimination learning separately, because retrieval of retrospective memories appears to be affected by training level, a matter considered later. Our concern in what follows is with the following phenomena: paradoxical discrimination learning, discrimination learning, resistance to extinction either in $\mathbf{S}+$ or in $\mathbf{S}-$ or both following discrimination training, and reversal learning.

Paradoxical discrimination consists of faster responding in $\mathrm{S}-$ than in $\mathrm{S}+$ in the early trials of discrimination learning. Consider first the initial trials of brightness differential conditioning. In these trials, the stimuli $S^{R}, S^{N}$, $\mathrm{S}+$, and $\mathbf{S}-$ have no explicit reinforcement history. Usually one brightness cue is $\mathrm{S}+$ for half the animals, with the other brightness cue being $\mathrm{S}+$ for the other half. Thus the exteroceptive cues are not a differential factor controlling performance in the early trials, but $S^{R}$ and $S^{N}$ are a different story. Many runway studies have reported that in the early trials of partial reinforcement, rats run faster following rewarded trials (i.e., in the presence of $S^{R}$ ) than following nonrewarded trials (i.e., in the presence of $\mathrm{S}^{\mathrm{N}}$; see, e.g., Capaldi, Wynn, \& Turner, 1962; McCain, Lee, \& Powell, 1962). Now consider a rat in the early trials of brightness differential conditioning running in the presence of the stimulus compounds $S^{R}, S-$ and $S^{N}, S+$. For some number of trials, the animal might manifest paradoxical discrimination, running faster in $\mathbf{S}-$ than in S+. Capaldi, Berg, and Morris (1975) varied the sequence of $\mathbf{S}+$ and $\mathbf{S}-$ trials, showing that paradoxical discrimination was due to rats' running faster when the stimulus compound was $S^{R}, S-$ as opposed to $S^{N}, S+$.

As for extinction, Capaldi et al. (1975) employed two groups, one of which received transitions from nonrewarded trials in S- to rewarded trials in S+ (Group 
S-S+) and one of which did not (Group S+S-). In Group $\mathrm{S}-\mathrm{S}+, \mathrm{S}^{\mathbf{N}}$ may be retrieved on a rewarded trial (in $\mathrm{S}+$ ), become a signal for reward, and so invigorate responding. This would not be the case for Group S+S-. Consistent with this analysis, subsequently when the two groups were extinguished in $\mathrm{S}+$ and in $\mathrm{S}-, \mathrm{S}+$ and $\mathrm{S}-$ trials being intermixed in extinction, Group $\mathrm{S}-\mathrm{S}+$ showed greater resistance to extinction than did Group $\mathbf{S}+\mathbf{S}-$.

Haggbloom (1980b, 1980c), employing brightness differential conditioning, has examined the effects of NR transitions from $\mathrm{S}-$ to $\mathrm{S}+$ in considerable detail. He has shown that such transitions retard not only extinction, but discriminative responding as well, by elevating Sspeeds. The latter effect has been named resistance to discrimination. In addition, Haggbloom has shown that the larger the reward magnitude contained in the NR transition from $\mathrm{S}-$ to $\mathrm{S}+$, the greater the resistance to both extinction and discrimination. Recall that in partial reinforcement, NL transitions produce greater resistance to extinction than NS transitions do.

In other investigations, partial reward has occurred in $\mathrm{S}+$, nonreward in $\mathbf{S}-$. In those investigations, groups that have received NR transitions within $S+$ have shown greater resistance to extinction and to discrimination and poorer reversal learning than groups that have not received such transitions (Haggbloom, 1980a, 1982).

There is a major difference between NR transitions that occur in S+ and those that occur from S- to S+. Those that occur within the same alternative are effective in the sense that $\mathbf{S}^{\mathbf{N}}$ stored on one trial is retrieved on the next trial, both early in training and late in training (e.g., Haggbloom, 1980a, 1982). However, those that occur from $S-$ to $S+$ appear to be effective in the sense described above only in the early trials of discrimination training (see especially Haggbloom, 1980a; Capaldi et al., 1991). In short, it appears that in the early trials of discrimination training, a memory stored in a given alternative will be retrieved on a subsequent trial in the other alternative. However, the tendency to retrieve in one alternative a memory stored in some other alternative declines as training progresses (see, e.g., Capaldi et al., 1991; Haggbloom, 1980a). In one sense this finding is not new. For example, it was demonstrated early on that as training progressed, a rewarded goalbox placement (or intertrial reinforcement) given in the transition between nonrewarded and rewarded trials lost its capacity to reduce resistance to extinction (see Capaldi \& Olivier, 1967). Presumably, as training progresses and as rats are better able to discriminate between, for example, the black and white alternatives (or an intertrial reinforcement trial and a runway trial), the tendency to retrieve memories in a situation other than the specific one in which they were stored declines. As we have seen, too, this is true when the runway and goalbox differ (e.g., Jobe \& Mellgren, 1974; Jobe et al., 1977) and when the storage ITI and the retrieval ITI differ (e.g., Capaldi et al., 1992; Capaldi et al., 1968; Capaldi et al., 1986b). Later in this paper, I shall speculate upon what memories are retrieved late in training in transitions from $\mathrm{S}-$ to $\mathrm{S}+$ and from $\mathrm{S}+$ to $\mathrm{S}-$.

Perhaps it should be mentioned that the sequential model was applied recently to what may be called an unorthodox form of discrimination learning-foraging in a radial maze (Roberts, 1991). Roberts baited different patches according to different reward sequences and found that rats were sensitive to these sequences and discriminated between patch locations on a sequential basis. Roberts was concerned with an interface between optimal foraging theory and psychological theory.

\section{MORE RECENT SEQUENTIAL DEVELOPMENTS: RESPONSE INTEGRATION AND EXPECTANCY}

One of the oldest and best known issues in animal learning theory is concerned with how the response is to be described, in terms of muscle movements or in terms of the effects on the environment such as getting the bar depressed (see, e.g., Lashley \& Ball, 1929; Muenzinger, 1928; Skinner, 1938; Tolman, 1932). It appears that both levels of description are useful for understanding learning and performance (see, e.g., Adams, 1984; Restle, 1957). Varied reward data are particularly relevant to two other issues concerning the response. One of these issues, which we will deal with in the section on new developments, concerns what sorts of associations are formed in instrumental learning (see, e.g., Colwill \& Rescorla, 1986). The third issue, which I will deal with in this section, concerns how and under what conditions elementary responses are organized into larger behavior patterns, which themselves function as units, forming the basis for still larger patterns of behavior (e.g., Capaldi, 1992).

One of the earliest and best answers to this issue was proposed by Skinner (1938). Skinner was concerned with how to conceptualize the response under a fixed ratio schedule of reinforcement. Under a fixed ratio schedule, a fixed number of barpresses-say, 10-must be completed in order to obtain a reinforcer. Skinner was concerned with how the response under the fixed ratio schedule might best be described-in terms of each individual press or in terms of the number of presses required to produce a reinforcer? Skinner opted for the latter approach, and considerable evidence indicates that the individual barpresses or pecks required to complete a fixed ratio come, with training, to function as a unit or chunk (see Capaldi, 1992, for a review of some of this literature). Interestingly, a number of early theorists (see Capaldi, 1992) have likened completing a fixed ratio to a trip down a runway. Let us accept the analogy as valid, assuming that the various behaviors involved in a runway "response," from orienting to the start door, to leaving the start box when the start door opens, to racing to the goalbox, to stopping over the goalcup and finally eating are fused, with training, into a new functional unit or chunk, which has its own properties. A major factor promoting how items are chunked is how the items are 
grouped. Grouping cues may be supplied by the experimenter, or they may be imposed on material by the learner (see, e.g., Crowder, 1976). An example of powerful grouping cues is the presentation of items either at the same spatial location or rhythmically (see, e.g., Bower, 1981). Many other types of stimuli have been employed as grouping cues (see Capaldi, 1992).

Capaldi (1992) referred to either the completion of a fixed ratio or a trip down the runway as a trial chunk. Is the trial chunk the highest level of organization of which rats are capable? According to Capaldi (1992), it is not. First, trial chunks may be combined into a higher unit of analysis called a series chunk. A series chunk is formed from two or more trial chunks. Series chunks may be organized in turn into list chunks. A list chunk is formed from two or more series chunks. Theorists, in speaking of responses, seldom explicitly indicate what level of organization they have in mind. For example, a variety of contemporary theorists have suggested that the primary association formed in instrumental learning is made between the response and the reward outcome, but none has explicitly indicated what is meant by "a response" (see, e.g., Colwill \& Rescorla, 1986; Mackintosh \& Dickinson, 1979). The problem with this lack of precision, as I see it, is this: It fails to recognize that determining how the events of a particular learning situation are organized into responses is indispensable to understanding learning and performance. As one example, consider how different expectancy theories, one emphasizing trial chunks (e.g., Amsel, 1958; Spence, 1960), and the other, series chunks (e.g., Capaldi, 1992), would react to a fixed repeating series of, say, four nonreinforced runway trials followed by a reinforced trial, an NNNNR series. Employing the trial chunk as the unit of analysis, the theorist would see expectancy of reward declining (extinguishing) progressively over the four nonrewarded trials, increasing on the rewarded trials. Thus, from the perspective of a trial chunk analysis, when a subsequent NNNNR series was presented, expectancy would be greatest on the first nonrewarded trial of the series. Employing the series chunk as the unit of analysis means that the NNNNR series of trials would be seen as a unit or chunk, an assumption I will justify in due course. Employing the series chunk as the unit of analysis, one would see expectancy of reward as low on the first nonrewarded trial of the series, increasing progressively over each successive nonrewarded trial, until by the rewarded trial, expectancy of reward would be very high. Following the rewarded trial, expectancy of reward would be low, in anticipation of the initial nonrewarded trial of the subsequent series. As may be seen, as concerns expectancy of reward, and thus vigor of responding, the emphasis on trial chunks leads to predictions diametrically opposed to those which follow from an emphasis on series chunks.

\section{Series Chunks}

Capaldi and Verry (1981) reported five pattern learning investigations which, taken collectively, indicate that rats have a far greater capacity than has ordinarily been realized to organize a series of discrete trials into a new functional unit or chunk, a series chunk. To better communicate the implications of the results of the Capaldi and Verry investigation and subsequent investigations from our and other laboratories, consider the following analogy. A person must employ several means of transportation in order to reach a destination in some remote area of the world. The list might go like this: take a car to the airport; take the plane; on landing, rent a car; drive to the boat dock and rent a boat; upon arrival up river, take a mule to the final destination. At any point in this series, the person is able to say which events have already occurred, if any; which events remain to occur, if any; and the specific order in which prior events have occurred and the order in which subsequent events are to occur. Moreover, this entire series of events can be used as a discriminative cue for another entire series of events. As an example of one series cuing another, the events of the two series might be recalled like this: and after I left Zambia, reaching my final destination by boat, by the way, I went to the Pyrenees, which involved, among other things, a bit of mountain climbing.

In Experiment 5 of Capaldi and Verry (1981), rats were given two slightly different five-trial series of rewarded and nonrewarded trials. The two series occurred in irregular order. Items of a series were separated by about $30 \mathrm{sec}$, series by about $15-20 \mathrm{~min}$. The items of the series were either a 20-pellet .045 -g reward (rewarded) or a 0 -pellet reward (nonrewarded). One of the five trial series consisted of a rewarded trial followed by four nonrewarded trials, RNNNN; the other consisted of four nonrewarded trials followed by a rewarded trial, NNNNR. The behavior of the rats under each of the two series is shown in Figure 1. Figure 1 shows running speed on each of the five trials of each series in blocks of 3 days. On the first few blocks, the rats ran pretty much the same in both series. One should realize that the rat had no way of determining whether Trial 1 was to terminate in reward or in nonreward, since the series were presented irregularly. Thus, the rats ran rapidly on Trial 1 in both series. However, given the Trial 1 reward outcome, the rats were in a position to correctly anticipate every subsequent event of each series. This they proceeded to do in a most revealing manner. First, the rats ran faster on each of the nonrewarded trials of the series terminating in reward than on the corresponding nonrewarded trials of the series terminating in nonreward. Moreover, while speeds on the nonrewarded trials of the series terminating in nonreward stayed slow by the final blocks, in the series terminating in reward, speeds increased steadily over Trials $2-5$, until by Trial 5 , they were very rapid. It is very difficult to understand the findings presented in Figure 1 without assuming that the animals both remembered and anticipated multiple reward outcomes. For example, consider the assumption that the animal remembered, say, only the first nonrewarded event of the NNNNR series. That assumption cannot possibly explain 


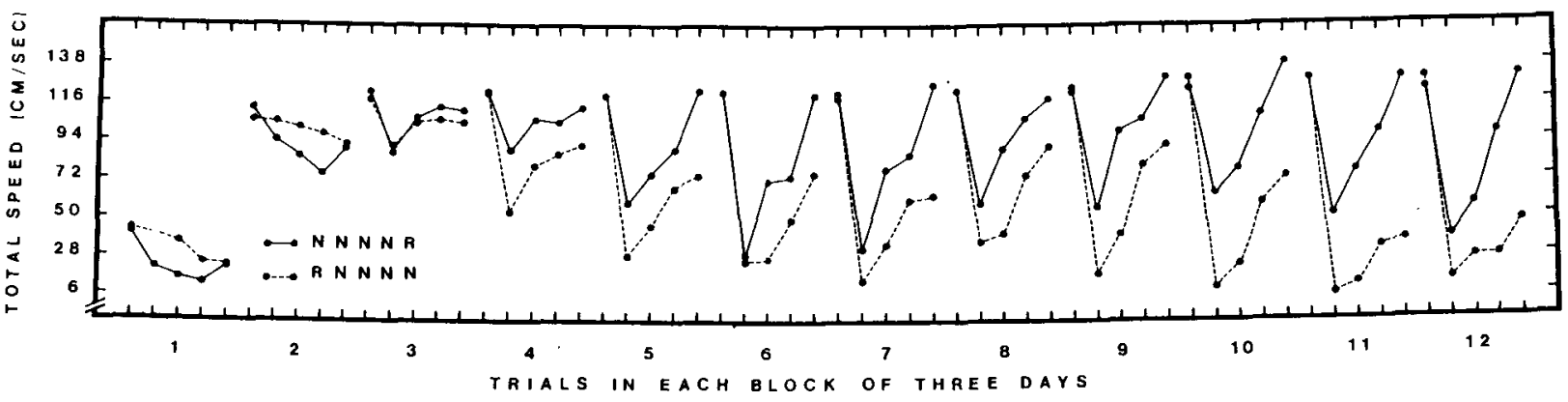

Figure 1. Running speed on each of the trials of the NNNNR series and the RNNNN series in blocks of 3 days.

fast running by Trial 5, because the memory of the first nonrewarded event also signaled nonreward on each of the Trials 2, 3, and 4. A variety of other hypotheses have been shown to be inadequate as an explanation of the findings shown in Figure 1 (see, e.g., Capaldi, 1985). The findings shown in Figure 1 strongly indicate that on each trial of each series the rats (1) correctly remembered each of the prior reward outcomes, employing that memory to correctly predict the current reward outcome, and (2) correctly anticipated each of the subsequent reward outcomes. For example, as early as Trial 2 of the NNNNR series, the animals were anticipating the terminal rewarded event as indicated by faster running on Trial 2 of the series terminating in reward than in the series terminating in nonreward.

The view of anticipation suggested in Figure 1 is considerably more complex than anticipation as it is conventionally understood. Conventional treatments emphasize anticipation of the reward outcome on the current trial. However, Figure 1 shows that the rats were anticipating not only the reward outcome on the current trial, but reward outcomes associated with subsequent trials as well, with responding on each current trial being influenced simultaneously by both sorts of anticipation. A theory of expectancy or anticipation suggested by these and similar data will be presented later in this paper.

\section{List Chunks}

If a series of trials is formed into a new functional unit, a series chunk, the series may have some of the characteristics of a single unitary event. That is, it seems possible that a series chunk might be able to give rise to a representation that might serve as a stimulus for some other series chunk. This was shown to be the case in a series of experiments reported by Capaldi et al. (1990), which were complemented and extended in a series of interesting investigations reported by Haggbloom, Birmingham, and Scranton (1992). In Experiment 2 of Capaldi et al. (1990), rats in a runway received two slightly different series of discrete trials, a rewarded trial followed by a nonrewarded trial-an RN series-and two rewarded trials followed by a nonrewarded trial-an RRN series. Trials of a series were separated by about a 15-sec ITI; series, by about a 15 -min interval. Consider, first, that if the rat was unable to determine which of the two series, $\mathrm{RN}$ or RRN, it was about to receive, it could not correctly anticipate all rewarded and nonrewarded trials. The major problem would arise in connection with Trial 2, a nonrewarded trial in one series (run slow) and a rewarded trial in the other series (run fast). However, if the rat had some way of determining which of the two series it was about to receive, it could respond correctly on every trial in each series, fast to reward and slow to nonreward. The rats were provided information about which of the two series was to occur and they did indeed anticipate every trial correctly, running fast and then slow in the RN series and fast, fast, and slow in the RRN series. What was the source of information? It was the same series, which had occurred 15 min earlier. That is, $\mathrm{RN}$ always preceded RN and RRN always preceded RRN, within a day. In the absence of this information, control rats were unable to correctly anticipate all events in both series (see Capaldi, 1992; Capaldi et al., 1990). Notice that in order to predict the current series correctly, the rat had to remember the entire prior series given 15 min earlier. The first trial of the RN and RRN series was the same-reward-and so could not serve as a discriminative cue for the next series. The last trial of the RN and RRN series was the same-nonreward-and so it too could not serve as a discriminative cue for the next series. We concluded that the animal remembered each of the series as a series, employing that series representation as a discriminative cue for the next series. We call this higher level of organization, employing a series of events as a cue for a series of events, a list chunk.

As indicated, there were many interesting ways in which Haggbloom et al. (1992) complemented and extended the findings of Capaldi et al. (1990). In Capaldi et al.'s finding described above, like series signaled like series (i.e., RN signaled RN and RRN signaled RRN). Among other things reported by Haggbloom et al. was that a given series could serve as a signal for an unlike series. In Experiment 2 of Haggbloom et al. (1992), rats correctly employed a reward, nonreward, reward series to signal a reward, reward, nonreward series or an RRN series (run fast, fast, slow) and an RNN series to signal an RNR series (run fast, slow, fast). Moreover, whereas Capaldi et al. employed time as a cue to group the series (e.g., 
RN, 15-min interval, RN), Haggbloom et al. grouped the series by employing a tactile cue. Capaldi et al. (1990) and Haggbloom et al. (1992) reported a variety of other findings relevant to list chunk formation, which the interested reader may find in the original sources and in Capaldi (1992).

It is clear on the basis of the results reported by Capaldi et al. (1990) and Haggbloom et al. (1992) that rats were able to determine which of the two series of events was about to occur prior to receiving the first trial of each series. They were able to do this on the basis of information extraneous to the series itself. The extraneous information may be a representation that characterizes either a prior similar series or a different series of events. Our view is that as training progresses, events are forged into increasingly higher order units or chunks. The event occurring within a trial becomes a unit, the trial chunk. Several trial chunks are forged into a unit, the series chunk. Several series are forged into a unit, the list chunk. The basis of such chunk formation is memory, a view briefly mentioned now and outlined in greater detail later. Within a trial, the animal is able to remember the succession of within-trial events leading to reinforcement. Within a series, the animal is able to remember the succession of trial outcomes leading to reinforcement. Within a list, the animal is able to remember the succession of series outcomes leading to reinforcement.

Central to our view is that animals remember not only prior events but the particular order in which those events occurred. Evidence that animals remember the order of events was supplied by Capaldi and Verry (1981; see also Haggbloom et al., 1992, Experiment 3). In Experiment 4 of Capaldi and Verry, rats received two slightly different series of trial outcomes in a runway-either reward, nonreward, nonreward (RNN) or nonreward, reward, reward (NRR). The series occurred irregularly, and events of a series were separated by about 15-30 sec, with series being separated by about $20 \mathrm{~min}$. With training, the rats came to run fast, slow, slow on Trials 1-3, respectively, of the RNN series and fast, fast, fast on Trials 1-3, respectively, of the NRR series. Examining the series will show that in order to respond discriminatively on Trial 3 of the series, fast in the NRR series, and slow in the RNN series, the animal had to remember the order in which the rewarded and nonrewarded trials occurred-that is, if $\mathrm{RN}$, run slow, and if NR, run fast. If the animal remembered merely the prior trial of the series, it could not have responded correctly on the current trial because, owing to the use of both series, reward was followed by both reward and nonreward and nonreward was followed by both reward and nonreward. Thus, to respond correctly on Trial 3, the animal had to remember two prior reward events and their order-if RN, then nonreward, and if NR, then reward (see also Yazawa \& Fujita, 1984).

\section{Counting}

There are many reasons for investigating numerical abilities in animals and, more specifically, as in our labora- tory, the ability of rats to enumerate or count successively presented goal events such as food reward and nonreward. Among the more obvious reasons is that of determining to what extent animals and humans may share in the complex cognitive capacities involved in counting. For the learning theorist, an additional, more subtle reason can be cited. Since representations of reward events regulate learning and performance, it becomes important to determine precisely what attributes or characteristics these representations might possess. For Hull and Sheffield (e.g., Sheffield, 1949), reward and nonreward gave rise to rapidly fading stimulus traces. For Amsel (1958), the goal-produced representation, seen as the feedback stimulus of classically conditioned goal reactions, may have emotional attributes such as anger or frustration. Wagner and Brandon (1989) have recently suggested that animals may be sensitive to the sensory attributes of reward events, hardness, and so forth - a view that is certainly supported by results obtained in our laboratory, as will become clear. For our part, as our investigations into the effects of $\mathrm{N}$-length on acquisition and extinction clearly suggest, we were and are concerned not only with the sensory and emotional attributes of reward events, but with abstract attributes of reward events rats might utilize as well, such as appreciating their number. As is perhaps clear from my work on N-length, I had long been of the opinion that rats were able to represent number of nonrewarded events by some means. I began to suspect more strongly that rats might be enumerating nonrewarded events when Verry and I (Capaldi \& Verry, 1981) found that they were highly accurate in correctly anticipating the rewarded trial of the NNNNR sequence as shown in Figure 1.

While we have investigated the capacity of rats to count successively presented nonrewarded events (Capaldi, Miller, \& Alptekin, 1988), we have focused on rewarded events for two reasons. Various $\mathrm{N}$-length findings already suggested that by one means or another, the number of nonrewarded events strongly controlled performance in acquisition and extinction; we wanted to demonstrate the same for the number of rewarded events. More importantly, counting can be investigated much more effectively by employing reward events rather than nonreward events. For example, counting investigations often require that two or more qualitatively different events be employed (e.g., count boys and girls), which is far easier to achieve with rewarded events than with nonrewarded events. In this connection, we have employed .045-g Noyes pellets and various cereals as rewarded events. As many as four qualitatively different rewarded events have been employed. Let us label these reward events A, B, $\mathrm{C}$, and $\mathrm{D}$.

According to Gelman and Gallistel (1978), counting involves the following. It consists of applying distinctive tags to events in one-to-one correspondence (in humans, numbers as $1,2,3, \ldots)$. Tags should be assigned in the same order over occasions-the stable order principle. Any event may be assigned any tag, so an order of presenting events is irrelevant-the order irrelevance prin- 
ciple. Finally, the tags must be abstract; the basis of tag assignment must be the number of events and not attributes such as size, shape, color, and so on. I have devised a situation in which rats have discriminated between the number of rewarded events in a manner consistent with the principles of counting suggested by Gelman and Gallistel (1978; see also Gallistel, 1990). In our situation, rats in a runway demonstrate counting by employing the number of successively presented rewarded events as a signal for a nonrewarded event (run slow).

The number of rewarded events may be confounded with a variety of other events that must be controlled or eliminated before one can conclude that rats count. To mention merely a few, as number of successively presented rewarded events in a runway increases, the following may increase: effort expended, distance run, elapsed time from the initial rewarded event to nonreward, amount eaten, time spent eating, number of responses, time spent waiting between trials, and still other variables. I certainly do not mean to suggest that these variables are ineffective in learning situations. Indeed, I would like to think that in addition to counting goal events, rats may also be capable of counting other events such as responses. Given the number and variety of events that may covary with the number of successively presented rewarded events, one might think that isolating the number of rewarded events as the controlling cue in our discrimination learning situation could be very difficult. Actually it has been relatively easy. The reason for this, in my view, is as follows. Rats are very adept at employing the number of rewarded or nonrewarded events as discriminative cues, or, to put it differently, cues associated with the numbers of rewarded and nonrewarded events are very salient to rats, much more salient than many other cues. Thus, the number cues associated with rewarded and nonrewarded events may gain greater control over responding than other cues that may covary with them; that is, number cues may overshadow a variety of other cues. This means that it is relatively easy experimentally to detect the influence of the number cues.

My view that rats are adept at counting (see Capaldi, 1993) contrasts strongly with that of Davis and Memmott (1982) and Davis and Perusse (1988) that rats count only as a last resort when no other alternative solution is available to them. My disagreement with Davis and his coworkers on this score precedes my explicit counting investigations and was based initially on my $\mathrm{N}$-length investigations. Explicit counting investigations have, in my view, confirmed the hypothesis, based on $\mathrm{N}$-length results, that rats are adept at counting. This assumption is important for the following reason. If rats count only as a last resort, then counting would clearly not be of much importance in understanding conventional learning investigations. The reason is straightforward: in conventional learning investigations, the number of rewarded and/or nonrewarded events is almost always confounded with one or more other variables that could serve as the basis of solution. Experiments from my laboratory clearly indi- cate, in my opinion, that the number of rewarded anc nonrewarded events is a highly salient cue for rats which may be employed even when other means of solution are available.

\section{The Counting Procedure}

Our basic counting procedure, of which there are variations, consists of presenting rats with two series of re warded and nonrewarded trials in irregular order in a run way. Trials of a series are separated by about $30 \mathrm{sec}$; series, by 10-30 min. One series might consist of two (or three) successive rewarded trials followed by a nonrewarded trial (i.e., RRN or RRRN). The other series differs only in that a nonrewarded trial precedes the rewarded trials (i.e., NRRN or NRRRN). Thus, a rat might be trained under the two series RRN and NRRN or RRRN and NRRRN. The typical finding from the use of such series is that, with training, the rats come to run fast on all trials except the terminal nonrewarded trial of each series. Sometimes speeds drop slightly over the rewarded trials, presumably in anticipation of this terminal nonrewarded trial. The procedure of presenting the rats with two slightly different series of trials in and of itself eliminates a number of alternative interpretations to counting rewarded events. Eliminated by the two-series procedure as signals for the terminal nonrewarded events, for example, are: the number of responses, time spent running, distance run, effort expended, elapsed time since Trial 1 of each series, and so on. In some other studies, after discriminative responding has occurred, we have varied the ITI between trials of a series and the time spent eating in the goalbox. These changes have had no effect on discriminative responding. Thus, these simple precautions have eliminated a number of other alternatives to counting rewarded events as signals for the terminal nonrewarded events, such as summing eating time, amount eaten, summing confinement time in the goalbox on rewarded trials, summing ITIs between rewarded trials, and so on.

The results obtained by employing the basic counting procedure described above appeared promising, so subsequent experiments were run which more firmly established that rats were indeed enumerating rewarded events. In devising these experiments, our strategy was relatively simple. We asked: Would rats enumerate events more or less as would people in similar circumstances? Consider, for example, presenting the following four-item series to people, using two different reward events, $A$ and $B$ : BAAN. What is the signal for nonrewarded? It could be the specific three events BAA. Or it could be the two rewarded events, AA. Or it could be three rewarded events of any kind, in which case three A events (or three B events) could be the signal for nonreward. It could be all of the above.

To determine how a rat might represent the BAAN series, Capaldi and Miller (1988a) provided rats each day with that series and a single nonrewarded trial $(N)$ in irregular order, the BAAN series and the nonrewarded trial 
being separated by about $10 \mathrm{~min}$. With training, the rats came to run fast on all trials except the terminal nonrewarded trial of the BAAN series. We then shifted half the rats to the series AAN and NAAN, the other half to the series AAAN and NAAAN. In shift, both groups ran fast on all trials except the terminal nonrewarded trials, and they did this after having received only one presentation of each series. These results indicate that the rats learned that the signal for the terminal nonrewarded trial was either two A events in succession or any three rewarded events of any kind, such as AAA. Let us consider these findings in some detail.

The findings described above are not due to nonspecific transfer effects. Burns and Gordon (1988) reported that, as in our experiment, rats trained BAAN and nonrewarded showed rapid transfer, to either AAN and NAAN or AAAN and NAAAN. However, Burns and Gordon reported that rats trained AAN and nonrewarded transferred poorly to AAAN and NAAAN, whereas rats trained AAAN and nonrewarded transferred poorly to AAN and NAAN. Moreover, the findings described above were replicated and extended under a variety of additional conditions (Capaldi \& Miller, 1988a, 1988b). For example, Capaldi and Miller (1988b) attempted to bias rats against counting to three under the BAAN series by training them with the additional series AAN. The hypothesis here was that by training rats under the AAN series they might employ only the two A events as the signal for the terminal nonrewarded event in the BAAN series. This was not the case. On being shifted to the AAAN and NAAAN series, the rats behaved appropriately almost immediately. And when subsequently shifted to AAN and NAAN, appropriate responding again appeared almost immediately. In another investigation (Capaldi \& Miller, 1988a, Experiment 6), rats trained BAAN and AAN showed immediate transfer to the series $A B B N$ and $B B N$.

The finding that rats trained BAAN and nonrewarded transfer almost immediately to either AAN and NAAN or AAAN and NAAAN suggests two more important points. Note, first, that in original training under the BAAN sequence, many cues simultaneously signaled nonrewarded, such as distance run; amount eaten; time elapsing in the goalbox, in the ITIs, etc.; number of responses; and others. Any of these cues could have been employed to signal the terminal nonrewarded event overshadowing number cues associated with successively presented rewarded events. This clearly was not the case. Distance run; number of responses; time elapsing between ITIs, in the goalbox, etc.; and many other variables as well could not be responsible for the transfer findings, because the values of such variables are different in the AAN and NAAN series and in the AAAN and NAAAN series. Moreover, these variables were confounded with the number of rewarded events in the original learning phase under the BAAN series. Despite such confounding, as the shift results show, the number of rewarded events nevertheless acquired control over responding. Thus the view that number cues associated with rewarded and nonrewarded events are highly salient to the rat (and perhaps other animals) and will be utilized even when other cues are valid is confirmed by the shift results for the BAAN series.

Experiment 7 of Capaldi and Miller (1988a) indicates not only that rats are capable of employing abstract number tags, but that they can apply the tags according to a complex same-different rule. In the original acquisition phase of that experiment, the signal for the terminal nonrewarded event was two rewarded events of the same kind. The same was true in transfer, except that novel reward events were employed. To be specific, in original acquisition the rats were trained under four different series-call them BAAN, ABBN, AAN, and BBN. Having learned to correctly anticipate the terminal nonrewarded event of all four series, the rats were shifted to the four series DCCN, CDDN, CCN, and DDN. Appropriate responding in transfer appeared immediately. It may be concluded that the rats learned the general rule: run slow after any two reward events of the same kind.

A variety of interesting but less dramatic counting findings have been reported. For example, it has been shown that rats trained simultaneously under the AAN and NAAN series (count to two) and the BBBN and NBBBN series (count to three) respond appropriately in all four series (Capaldi, Miller, \& Alptekin, 1989). Burns and Sanders (1987) reported that rats given, for example, AAN and NAAN in, say, a white runway (count to two) and AAAN and NAAAN in, say, a black runway (count to three) respond appropriately in all four series. The results show that rats can conditionalize their count on the item being counted, A or B (Capaldi et al., 1989), and on external cues, a white or a black runway (Burns \& Sanders, 1987).

\section{Serial Learning}

A frequently employed procedure for investigating serial learning in animals is the following. Rats in some apparatus, frequently a runway, are presented in succession with a series of different reward magnitudes in a fixed order, the terminal event of the series often being nonreward (see, e.g., Fountain, 1990). In this approach to serial learning, which I shall call reward magnitude serial learning, rats may with training come to anticipate the terminal nonrewarded event correctly. The reward magnitude serial learning literature has recently been reviewed in detail by Compton (1991). Besides reward magnitude serial learning, other types of animal serial learning investigations have been reported. These have employed as items stimuli other than reward magnitudes (e.g., geometric forms), and in some cases, items have been presented simultaneously rather than successively (see, e.g., D'Amato, 1991; Terrace, 1991; Wright, 1992). While these studies are interesting and important, our focus here is on reward magnitude serial learning.

The original and to some extent the sustaining impetus to reward magnitude serial learning is the view that rats encode the rule structure of a series (e.g., Hulse \& Dorsky, 1977). The close identification between reward 
magnitude serial learning and rule learning raises two major issues. One is this: To what extent are findings in connection with reward magnitude serial learning consistent with the rule learning view, or with other views subsequently offered to explain reward magnitude serial learning, the position-item view (e.g., Roitblat, 1982; Roitblat, Pologe, \& Scopatz, 1983), and the sequential view (e.g., Capaldi \& Molina, 1979)? The second issue has to do with exploring the implications of the view expressed by Hulse (1980) that there are "boundaries between learning situations conducive to reward memories and $\mathbf{S}-\mathrm{S}$ or $\mathrm{S}-\mathrm{R}$ associative learning and those conducive to information processing and rule learning"' (p. 690). The first issue, which has been extensively debated in the literature, will be treated only in broad outline here. The second issue, which has not been examined previously at any length, will be covered in some detail in relation to the position-item view as well as rule learning.

A major implication of the rule learning view is that the simpler the rule structure of a series, the faster it will be learned. By 14-7-3-1-0, I denote the number of .045-g food pellets received by rats on each of five successive trips down a runway. The 14-7-3-1-0 series may be described as a strongly monotonic series, and therefore as a relatively simple series characterized by a single rule. The 14-1-3-7-0 series, a nonmonotonic series, is more complicated than the 14-7-3-1-0 series, since items decrease as well as increase. Consistent with rule learning, the 14-7-3-1-0 series was learned faster than the 14-1-3-7-0 series (Hulse \& Dorsky, 1977). However, one problem for the rule learning view is that many of the findings said to support it can also be explained by other views. A second problem is that many findings inconsistent with rule learning have been reported. Let us first consider alternative interpretations of data said to support rule learning.

According to the sequential hypothesis, of course, rats learn associations between the items of a series. According to the position-item view, as advocated by Roitblat (1982) and Roitblat et al. (1983), rats do not learn associations between the items of a series but rather rats learn associations between an item and its position in the series. The position-item view, of course, was first suggested in connection with human serial learning data (see, e.g., Crowder, 1976) and has been extensively employed to explain various other forms of animal serial learning, as, for example, when items such as geometric forms are presented simultaneously to monkeys or pigeons (see, e.g., D'Amato, 1991; Terrace, 1991). In any event, the position-item view can explain faster tracking of the 0 -pellet item in the 14-7-3-1-0 series than in the 14-1-3-7-0 series by assuming that (1) stronger excitatory tendencies exist at Position 4 in the nonmonotonic series (7-pellet reward) than in the monotonic series (1pellet reward); (2) greater generalization of excitation from Positions 4 to 5 occurs in the nonmonotonic series than in the monotonic series, and (3) rats will therefore run faster on Position 5 in the nonmonotonic series than in the monotonic series.
The sequential hypothesis, like the position-item view, can appeal to stimulus generalization to explain reward magnitude serial learning. The immediate signal for 0 pellets is the memory of 7 pellets in the nonmonotonic series and 1 pellet in the monotonic series. The memory of 3 pellets is most similar to the memory of 7 pellets in the nonmonotonic series and to the memory of 1 pellet in the monotonic series. Thus, any excitatory capacity acquired by the memory of 3 pellets would generalize strongly to the memories of 7 pellets and 1 pellet. The memory of 3 pellets acquires more excitatory capacity to evoke responding in the 14-1-3-7-0 series, where it occurs on 7-pellet trials, than it does in the 14-7-3-1-0 series, where it occurs on 1-pellet trials. Thus, the memory of 3 pellets supplies more generalized capacity to elicit responding to 7 pellets in the nonmonotonic series than it supplies to 1 pellet in the monotonic series. Thus, running should be faster to 0 pellets in the monotonic series than in the nonmonotonic series.

A variety of other findings said to support rule learning are also amenable to interpretation in terms of sequential associations between item memories. As one example, two groups of rats, a monotonic group trained 14-7-3-1 and a nonmonotonic group trained 14-3-7-1, were subsequently shifted to $14-7-3-1-0$ and 14-3-7-1-0, respectively (see, e.g., Fountain \& Hulse, 1981). The added 0 -pellet item was anticipated better (i.e., running was slower) in the monotonic group than in the nonmonotonic group, supporting the rule encoding view. However, as is perhaps clear, item memories can also explain this finding. Briefly, the memory of 1 pellet, which is the signal for 0 pellets in both series, would receive more generalized associative strength from the similar memory of 3 pellets in the nonmonotonic series, where it occurs on 7-pellet trials, than in the monotonic series, where it occurs on 1-pellet trials. Other examples of how sequential memories can explain findings which are also consistent with a rule learning view have been provided elsewhere (see, e.g., Capaldi, 1992).

A variety of findings have been reported which are consistent with a memory discrimination learning approach to reward magnitude serial learning while being inconsistent with both rule learning and the position-item view (see, e.g., Capaldi, Nawrocki, Miller, \& Verry, 1985; Haggbloom, 1985). I consider in detail only one example of this. Haggbloom (1985) trained four groups of rats under the five-trial series 14-7-3-1-0. After anticipation of the 0-pellet item appeared, the rats were shifted to one of four test series: $20-14-7-3-0$, a five-trial monotonic series; 20-7-3-14-0, a five-trial nonmonotonic series; 20-14-7-3-1-0, a six-trial monotonic series; and 20-714-3-1-0, a six-trial nonmonotonic series. The rule learning view predicts that the five- and six-trial monotonic groups should perform better in transfer than the five- and six-trial nonmonotonic groups, respectively. The positionitem view predicts that the five-trial groups should perform better in transfer than the six-trial groups because that position was nonrewarded in original learning. The sequential view predicts that the two six-trial groups should per- 
form better in transfer than the two five-trial groups, because in these groups the memory of 1 pellet signals 0 pellets in transfer as in acquisition. The transfer findings were consistent with the predictions of the sequential view.

Is reward magnitude serial learning unique? Procedurally, reward magnitude serial learning is similar to a variety of varied reward tasks, such as varied magnitude of reward, partial reward, and discrimination learning. Similarity extends beyond procedure; my coworkers and I have shown, for example, that variables (e.g., NR transitions) that have particular effects on performance in various varied reward situations have identical effects in reward magnitude serial learning situations (see, e.g., Capaldi et al., 1986b; Capaldi, Verry, Nawrocki, \& Miller, 1984). It would seem reasonable to suggest, therefore, that any theory which purports to explain reward magnitude serial learning should also be required to explain various other varied reward data or vice versa. This, of course, is the approach adopted by me and my coworkers. Essentially we have argued that while reward magnitude serial learning data may be interesting, they are in no sense unique (see especially Capaldi et al., 1984).

However, it has been explicitly argued by Hulse (1980) that reward magnitude serial learning, properly defined, is a unique form of learning. According to Hulse, reward magnitude serial learning is to be explained in terms of rule learning and information processing, whereas other varied reward phenomena may be explained, as the sequential view has explained them, in terms of associations between memories of items and items ( $\mathrm{S}-\mathrm{S}$ associations) and between memories of items and the instrumental response (S-R associations). What separates reward magnitude serial learning from conventional varied magnitude situations according to Hulse? Hulse suggested that animals encode rules under the following conditions: the series consists of three or more different reward magnitudes; the interval between items of the series is short, $10-15 \mathrm{sec}$ rather than, say, $5 \mathrm{~min}$; the interval between series presentation is relatively short, say, $15 \mathrm{~min}$, rather than $24 \mathrm{~h}$; there should be more than one presentation of the series each day, say, four; and extensive training must be given. According to Hulse, the conditions outlined above facilitate comparisons between items of a series thus enabling animals to encode rules. For example, according to Hulse, if a series contains only two different reward magnitudes, the rule that these items may describe may be so impoverished "that they no longer fairly and clearly represent serial patterns at all" (p. 689).

Early studies from my laboratory which were inconsistent with the rule learning view (e.g., Capaldi, Blitzer, \& Molina, 1979; Capaldi \& Molina, 1979) employed procedures which Hulse (1980) subsequently suggested were inappropriate for examining rule learning. Subsequently, however, the rule learning view was disconfirmed by employing conditions which, according to Hulse (1980), were favorable to rule encoding (see, e.g., Capaldi, Nawrocki, Miller, \& Verry, 1986a; Capaldi et al., 1984; Haggbloom, 1985; Haggbloom \& Brooks, 1985). For example, it has been shown several times, employing conditions said to be favorable to rule learning (as well as not), that rats may learn nonmonotonic and weakly monotonic series more rapidly than strongly monotonic series (e.g., Capaldi \& Molina, 1979; Capaldi et al., 1985; Haggbloom, 1985; Haggbloom \& Brooks, 1985). These results were shown to be consistent with the memory-discrimination learning approach suggested by the sequential view and thus were taken to suggest that reward magnitude serial learning, even as defined by Hulse (1980), is not a unique form of learning and should be seen as similar to various other varied reward phenomena. Below, I wish to examine other reasons for regarding reward magnitude serial learning and various varied reward phenomena as similar. These reasons have not been previously considered in print.

My general point below is that the rule learning view and, as I will show subsequently, the position-item view are faced with the following dilemma. On the one hand, the argument that reward magnitude serial learning and other varied magnitude situations differ fundamentally is implausible. On the other hand, seeing them as similar creates problems, since both views are inconsistent with a wide variety of varied reward data. The nature of the dilemma could be illustrated by employing a number of different varied reward phenomena. I will attempt to do so initially by employing the effects of single alternation reward schedules on acquisition and extinction.

Consider rats that manifest single alternation pattern learning at a short ITI-say, 15-30 sec. Have they learned a rule-specifically, the rule that reward and nonreward trials alternate? Perhaps not according to Hulse (1980), since in his view series with only two reward events do not provide sufficient information for the proper rule to be unambiguously encoded. Can we take seriously a view that emphasizes rule learning but suggests that animals may not encode the simple rule that rewarded and nonrewarded trials alternate? Consider, too, that very complicated rules can be generated from a mere two events (e.g., they may be used to construct a counting schedule, such as AAN and NAAN; see, e.g., Capaldi \& Miller, 1988a). I think it is implausible to maintain that animals learn rules but may not learn the simple rule that rewarded and nonrewarded events single alternate. For the sake of argument, let us accept that rats learn the alternation rule under some conditions deemed favorable to rule encoding by Hulse (1980)-for example, at short ITIs. The problem with this approach is that rats alternate at long as well as at short ITIs (see, e.g., Jobe et al., 1977). Hulse (1980) might suggest that rats learn the alternation rule at short but not at long ITIs. The problem with this formulation is that available data reveal that variables which facilitate single alternation pattern running at short ITIs also facilitate it at long ITIs (see Jobe et al., 1977) and that other sequential effects are similar at all ITIs (e.g., Capaldi \& Capaldi, 1970; Jobe \& Mellgren, 1974). 
Roitblat and his coworkers (Roitblat, 1982; Roitblat et al., 1983) have applied the position item view to reward magnitude serial learning but not to varied reward data generally. D'Amato (1991) has suggested, however, that the position-item view may be applied to varied reward data. This suggestion is not feasible in my view. For example, the position-item view seems implausible when applied to data reported by Bloom and Capaldi (1961), who trained rats under a single alternation schedule with 12 trials held each day. They reported that the rats eventually ran faster on all rewarded trials than on all nonrewarded trials. Are we to believe that rats are capable of discriminating between Position 11, a rewarded trial run fast, and Position 12, a nonrewarded trial run slow? Even if rats were able to discriminate between the 11 th and 12 th positions in a series, consider too that we have found in our laboratory (unpublished study) that rats will show single alternation patterning when on half the days the series begins with a rewarded trial and on half the days it begins with a nonrewarded trial. When trained in this way, the rat runs fast on Trial 1 of the day $(50 \%$ reward). Following Trial 1, the rat behaves in accordance with the single alternation series on all subsequent trials; that is, on Trial 2, it runs fast if Trial 1 was nonrewarded, and slow if Trial 1 was rewarded. Under these conditions, of course, all positions in the series are partially rewarded, and because there is no consistent relation between position and reward event, single alternation patterning should be impossible, according to the position-item view. This finding should not be surprising: my coworkers and I have reported similar findings often. For example, in Experiment 4 of Capaldi and Verry (1981), which was considered previously, rats correctly anticipated rewarded and nonrewarded trials which occurred on Trials 2 and 3 of their series when trained concurrently under the two series RNN and NRR, an impossibility if all that rats learned were position-item associations. Too, my counting studies described earlier cannot be explained in terms of positionitem associations. In the series AAN and NAAN, Position 3 received $50 \%$ irregular reward, for example, yet rats ran slow on Trial 3 of AAN and fast on Trial 3 of NAAN.

\section{A Future Direction for Reward Magnitude Serial Learning}

In animal serial learning, as in human serial learning, series may be employed, and often are, which are confounded in the sense that any result obtained may plausibly be explained by two or more different theories. Crowder (1976) provides examples of such confounding in human serial learning. An example of such confounding in animal serial learning was supplied earlier, when it was shown that faster learning of the monotonic series 14-7-3-1-0 than of the nonmonotonic series 14-1-3-7-0 could be explained by three different views: rule learning, position-item associations, and the memory sequential approach. Let us examine this current controversy in the light of an earlier one in connection with maze learning by rats.

Early on there was considerable controversy concerning the sensory basis of maze learning in rats. Did rats learn mazes by employing vision? Or smell? Or kinesthesis? And so on. A considerable number of investigations ultimately revealed that rats could learn mazes by employing any of these sensory avenues (see especially Munn, 1950). If rats were denied, say, vision, either by blinding them or by running them in total darkness, they would nevertheless learn the maze employing, say, kinesthesis. In well-illuminated rooms with plenty of landmarks, however, vision appeared to be dominant (see, e.g., Munn, 1950; Restle, 1957). I think that the controversy in reward magnitude serial learning resembles in many ways that which occurred in the early days of maze learning. As one example, demonstrating that rats master series impossible to learn on the basis of position-item associations (e.g., the RNN and NRR series) does not mean rats are incapable of employing such associations. I do think that rats have some limited capacity to learn positionitem associations when these are valid. For example, I do not doubt that rats can discriminate between Positions 1 and 2 of a series. However, rats may not be able to discriminate between, say, Positions 11 and 12 of a series.

I also think that rats learn rules. I have said as much, it will be recalled, in connection with my counting studies. I suggest that rats given a single alternation schedule will encode the rule that rewarded and nonrewarded trials alternate, if they are given sufficient training-say, about 80-100 trials (see, e.g., Bloom \& Capaldi, 1961). Early in training under the single alternation schedule, rats learn associations between memories of items and items and between memories of items and the response. Forming such associations is in no way incompatible with learning rules. The difference between my position and Hulse's is this: he suggests that the conditions under which animals learn S-S and S-R associations preclude rule learning and vice versa. I do not see a necessary incompatibility between associative learning and rule learning. I would agree with Hulse, however, that rule learning emerges later in training than associative learning, at least in the naive animal.

An implication of the position expressed above is that some sorts of research are not as useful for making inferences as they might otherwise seem. Specifically, demonstrating that serial learning occurred under conditions impossible to explain by theory $X$ does not necessarily imply that theory $X$ is useless and should be rejected. It may be that theory $X$ is a viable explanation of serial learning under some conditions. If so, our approach should be to discover the conditions, if any, under which theory $X$ plays a role in explaining behavior. This approach, if adopted, would contrast with that so far employed in reward magnitude serial learning, which has emphasized theory testing and rejection. What I am suggesting here is hardly new. As early as 1913, it was suggested that 
in serial tasks people might learn item-item associations, position-item associations, and rules, and might organize series into chunks (see Woodworth, 1938).

\section{A NEW DIRECTION: A THEORY OF EXPECTANCY}

Consider an animal that is about to respond on trial $X$. According to conventional usage, if on trial $X$ the animal remembers the reward event that occurred on, say, the previous trial, it is employing retrospective memory. And if on trial $X$ the animal anticipates the reward event contingent on its current response, it is employing what some call prospective memory (e.g., Chatlosh \& Wasserman, 1992), more commonly called anticipation or expectancy (e.g., Amsel, 1992). The sequential view has emphasized retrospective memory, pointing to what it considered to be inadequacies of theories that emphasize prospective memory. Historically, of course, prospective memory or anticipation has been much more heavily emphasized than retrospective memory in animal learning, to say the least (see, e.g., Amsel, 1958; Hull, 1930; Spence, 1956; Tolman, 1932). A new and promising direction for the growth of the sequential view is the utilization of an expectancy assumption. The incorporation of an expectancy assumption into the sequential view will allow it to explain additional phenomena, phenomena not explained by other views, and will suggest new directions for further research. In what follows, I will, first, describe in general terms the approach to expectancy utilized by the sequential view, an approach that is in some respects unique and in other respects quite conventional. Second, I will describe the specific assumptions employed by the present approach to expectancy. Finally, employing the present sequential expectancy approach, I will explain a variety of phenomena that until now have proved perplexing to all types of theories.

\section{Expectancy: A General Description of the Sequential Approach}

Two major differences exist between the sequential view of expectancy and other views. One difference, stated informally, is this. Traditional views emphasize, and emphasize only, what the animal may be expecting on the current trial $X$. On the basis of the sort of data I described in the previous section, I suggest that the animal may come to expect not only the reward event to occur on the current trial $X$, but that associated with one or more future trials $X+1, X+2$, etc. For example, an animal given the two series of rewarded and nonrewarded trials, RNR and RNN, in regular order will eventually come (1) to anticipate the Trial 2 event, nonreward, in both series (run slow), but (2) will run more slowly on that trial in the series terminating in nonreward, the RNN series, than in the series terminating in reward, the RNR series (see, e.g., Capaldi, 1992; Capaldi \& Miller, 1988c; Capaldi, Nawrocki, \& Verry, 1983; Capaldi \& Verry, 1981). Such behavior suggests that on Trial 2 the animal is anticipat- ing not only the Trial 2 event, nonreward, but also the Trial 3 event, running faster on Trial 2 when that event is reward (RNR series) than when that event is nonreward (RNN series). Let us call this multitrial expectancy. The basis of multitrial expectancy is chunking. As indicated earlier, rats form not only trial chunks, emphasized by traditional theories, but series chunks, emphasized by the present view. Series chunks are the basis of multitrial expectancy.

The very meaning of expectancy is that some event which occurs prior to, for example, a reward event, will give rise to a representation of that reward event. In traditional formulations, the events giving rise to expectancy tend to be identified as exteroceptive cues such as visual or auditory cues, although cues associated with food deprivation and the like have also been implicated (see, e.g., Hull, 1931), and more recently, Lobaugh, Greene, Grant, Nick, and Amsel (1989) have suggested that stimulus traces may give rise to expectancies. I differ with the traditional view in two main ways. First, I think that too great a role is assigned to exteroceptive stimuli in promoting expectancy. Second, I think that the traditional approach overlooks, or at least minimizes, a major source of stimulation that gives rise to expectancies - that associated with various retrospective memories. Others have also suggested that animals employ both retrospective and prospective memory (see, e.g., Chatlosh \& Wasserman, 1992; Wasserman, 1986). Moreover, within the framework of anticipatory response theory, which heavily emphasizes prospective memory, a mechanism of retrospective memory is to be found: a fading stimulus trace. Unconditioned responses such as primary frustration $\left(\mathbf{R}_{\mathbf{F}}\right)$ produce stimuli $\left(\mathrm{S}_{\mathrm{F}}\right)$ that may persist briefly and acquire control over responding (see, e.g., Amsel, 1958, 1962, 1992).

Other views which have suggested that behavior is controlled by both retrospective and prospective memories have seen each as exercising independent control. I am suggesting that the two operate in tandem-that retrospective memories give rise to prospective memories. How does this sort of S-S association differ from one in which, say, a visual cue elicits the expectancy? Only in this: a retrospective memory, rather than, for example, a visual cue, elicits the expectancy. This assumption is in one respect quite conventional. That is to say, once it is granted that reward events occur contiguously with retrospective memories, there is no way to avoid the assumption that retrospective memories may come to elicit prospective memories exactly as any other stimuli do. In one respect, all I am suggesting is that a class of stimuli not previously seen as giving rise to expectancies actually does so.

Before proceeding with a specific analysis of how and when retrospective memories may come to elicit prospective memories, let me briefly describe the possible internal state of an animal on a particular trial $X$, according to the view being advanced here. First, on some trial $X$ the animal may remember, retrospectively, reward events associated with one, two, or more prior trials and the 
order in which those events occurred. Too, as indicated above, on trial $X$ the animal is capable of remembering, prospectively, not only the reward outcome associated with trial $X$ but outcomes associated with one or more subsequent trials, trial $X+1$, trial $X+2$, etc. Thus, according to the retrospective-prospective view, on a given trial $X$ the animal may be remembering, both retrospectively and prospectively, a variety of reward events, its behavior being influenced by both sources of memory (see Figure 1).

\section{The Sequential View of Expectancy: Specific Assumptions}

I assume that, with training, expectancies come to reflect the reward conditions actually in force in connection with a particular stimulus. As one example, if a particular stimulus is associated with reward on half the occasions and nonreward on the other half, the animal will form the expectancy that "reward and nonreward occur about equally often." As another example, if a particular stimulus is associated with large reward, the animal will form an expectancy of larger reward. I refer to this as the content of the expectancy.

Expectancies have strength as well as content. The capacity of a given stimulus to give rise to an expectancy of a certain content is an increasing function of the number of conditioning trials. Thus, a given stimulus may have a strong capacity to give rise to an expectancy of small reward. Or it may have a weak capacity to give rise to an expectancy of large reward. A question arises: Under what conditions does a stimulus acquire the capacity to elicit a particular expectancy?

In instrumental learning situations, the reward events contingent upon an instrumental response are preceded by a variety of cues. We know from both Pavlovian and instrumental investigations that the predictive value acquired by each of these cues will depend on both its saliency and its validity relative to other cues (e.g., Dickinson, 1980; Diez-Chamizo, Sterio, \& Mackintosh, 1985; Haddad, Walkenbach, Preston, \& Strong, 1981; Haggbloom, 1981; Wagner, 1971). Given such findings, I suggest, perhaps unsurprisingly, that the cue or cues which most validly predict a given reward event, reward or nonreward, will give rise, all else being equal, to the strongest expectancy of that reward event. The cue that most validly signals a reward event may be of any kind. It may be an exteroceptive cue. It may be an interoceptive cue. More specifically, the interoceptive cue may be the prospective memory of one or more reward events. Below I will show that in, say, partial reward situations, prospective memories of reward events cannot be less valid than static apparatus cues and that in general they are more valid, sometimes much more valid than apparatus cues. I will illustrate this by using initially a regular reward schedule, a single alternation schedule, and then an irregular $50 \%$ schedule.

Under the single alternation schedule, the runway apparatus cues are followed by reward $50 \%$ of the time and by nonreward $50 \%$ of the time. Thus, the apparatus cues are much less valid predictors of reward and nonreward than the retrospective memories are. That is, the apparatus cues can tell the animal only that reward may occur on this trial with a probability of .50 . However, the retrospective memory of nonreward, or $S^{N}$, is a perfectly valid predictor of reward, a $100 \%$ predictor. And the retrospective memory of reward, $\mathrm{S}^{\mathrm{R}}$, is a perfectly valid predictor of nonreward, a $100 \%$ predictor. Let us label the expectancies of reward and of nonreward as $E^{R}$ and $\mathrm{E}^{\mathrm{N}}$, respectively. Under the single alternation schedule, then, we would have the following $S-S$ associations: $S^{N}$ would elicit $E^{R}\left(S^{N} \rightarrow E^{R}\right)$, and $S^{R}$ would elicit $E^{N}\left(S^{R} \rightarrow\right.$ $\mathrm{E}^{\mathrm{N}}$ ). Put informally, when later in training $\mathrm{S}^{\mathrm{N}}$ is retrieved, the animal anticipates reward and so runs fast, and when $S^{R}$ is retrieved, the animal anticipates nonreward and so runs slow. Let us consider now an irregular 50\% schedule of reward.

Recall that rats are capable of determining, within limits, how many nonrewarded or rewarded trials have occurred. When this is the case, a given retrospective memory cannot be a less valid signal for some reward event than the static exteroceptive cues can. By way of example, let the overall percentage of irregular reward be $50 \%$ and let the percentage of reward associated with a particular retrospective memory be $30 \%, 50 \%$, or $70 \%$. For example, the schedule may be so constructed that a single nonrewarded trial (or rewarded trial) is followed by a rewarded trial on $30 \%, 50 \%$, or $70 \%$ of the occasions. In the $30 \%$ case, the particular retrospective memory will be a more valid predictor of nonreward than the static apparatus cues will. In the $50 \%$ case, both the particular retrospective memory and the static apparatus cues will be equally valid predictors of reward and nonreward. In the $70 \%$ case, the particular retrospective memory will be a more valid predictor of reward than the static apparatus cues will. Consider also that irregular schedules are constructed such that only a given number of rewarded or nonrewarded trials is allowed to occur in succession, say three. Under such a schedule, the retrospective memory associated with three $R$ trials in succession, call it $S^{R 3}$, will be a perfectly valid signal for nonreward $\left(S^{R 3} \rightarrow E^{N}\right)$, and the retrospective memory associated with three nonrewarded trials in succession, $\mathrm{S}^{\mathrm{N} 3}$, will be a perfectly valid signal for reward $\left(\mathrm{S}^{\mathrm{N3}} \rightarrow \mathrm{E}^{\mathrm{R}}\right)$. Because of retrospective memory, then, even in a so-called $50 \%$ irregular schedule of partial reward, the animal could find itself in a position to correctly predict reward outcomes on a better than chance basis, and this could be the case much more often than not, depending on the specific arrangement of rewarded and nonrewarded trials in the schedule.

As implied in the preceding comments, better expectancies-those associated with larger magnitudes or higher reward percentages-elicit more vigorous responding. This may occur, obviously enough, via a motivational route. It may also occur via a conditioning route. As an example of the latter, recognize that larger reward not only results in a "better" expectancy, but it results in a 
stronger association between $\mathrm{E}^{\mathrm{R}}$ and the instrumental response; that is, all stimuli including $S^{R}$ and $S^{N}$ are more strongly conditioned to, the larger the reward magnitude. An example of the associative structure I have in mind, then, is as follows: $S^{R}$ elicits $E^{R}$, an $S-S$ association, and both $S^{R}$ and $E^{R}$ elicit the instrumental response, an $S-R$ association. Except for the prominent role assigned to $S^{R}$, the associative structure identified above is in many respects conventional (see, e.g., Amsel, 1992).

\section{Sequential Expectancy: Explaining \\ Some Perplexing Phenomena}

The view of reward expectancy outlined above allows the sequential model to explain a variety of additional phenomena. As an illustration of this, I will apply the sequential expectancy model to a variety of phenomena that have existed in the literature for many years without having been adequately explained, despite their being of critical theoretical significance. These phenomena are the effects of overtraining on extinction following partial reward, the effects of overtraining on acquisition responding under a $50 \%$ irregular schedule of partial reward, and the effects on extinction of a shift from a single alternation schedule of partial reward to a schedule of consistent reward.

Overtraining and partial reward extinction. It has been shown, employing partial reward, that extended acquisition training, called overtraining, may result in reduced resistance to extinction relative to smaller numbers of acquisition trials. This was shown employing a single alternation schedule of partial reward (Capaldi, 1958) and a 50\% irregular schedule of partial reward (McCain et al., 1962; Wagner, 1963). These extinction findings were shown to be inconsistent with Amsel's (1962) frustration hypothesis by Wagner (1963) and indeed were suggested to be incompatible with any available hypothesis by McCain et al. (1962). While Capaldi (1958) and McCain et al. (1962) employed a very short ITI, 15-30 sec, that employed by Wagner (1963) was not explicitly specified but it was undoubtedly longer. In any event, it may be that the effects of partial reward overtraining on extinction is a massed trial effect. Daly and Daly (1982) have considered the effects of overtraining on extinction relative to $50 \%$ irregular reward. The present explanation of the overtraining partial reward effect is as follows. As a result of overtraining, rats come to learn better and better the contingencies built into even a $50 \%$ irregular schedule of reward, much less a single alternation schedule. Most importantly, they learn, with some degree of precision, what the longest $\mathrm{N}$-length in acquisition is and, as indicated above, following that number of nonrewarded trials, expectancy of reward is high. Overtraining thus introduces two new factors in extinction as opposed to more limited training. First, it aids discrimination of the acquisition conditions from the extinction conditions in that the animal is better able to determine when the number of nonrewarded trials in extinction exceeds the longest $\mathrm{N}$-length in acquisition. Second, when this occurs, it results in the nonreinforcement of a strong expectancy of reward and thus, as Amsel (1958, 1962) has suggested, in considerable disappointment or frustration. Together, these two factors-enhanced discrimination and frustrationwould hasten extinction.

Overtraining and partial reward in acquisition. Figure 1 of this report, as well as other findings from my laboratory (see, e.g., Capaldi, 1964, 1966, 1992), indicate that rats are able to determine with some precision how many nonrewarded trials precede a rewarded trial. Too, my counting studies show that rats are able to determine with some precision how many rewarded trials precede a nonrewarded trial (e.g., Capaldi \& Miller, 1988a). Both of these findings were obtained employing highly regular fixed repeating schedules. Is there any reason to assume that rats are sensitive to similar, but more subtle contingencies, when they are presented in an irregular $50 \%$ schedule of reward? Fortunately, for the present view of expectancy, there is. In McCain et al.'s (1962) investigation, which employed either 60 or 200 trials of irregular $50 \%$ reward, the probability of reward following reward was .34 and the probability of reward following nonreward was .62. Early in training-say, Trials 1-60-the rats tended to run faster following reward than following nonreward-a common finding, as will be recalled. From about Trials $80-140$, running was about equally fast following rewarded and nonrewarded trials. But by the end of training, on Trials 181-200, running tended to be faster following nonrewarded than following rewarded trials. As one index of this, early in training (Trials $1-10$ ), it appears that only 1 of 18 rats ran faster following nonrewarded than following rewarded trials, whereas by the end of training (Trials 181-200), 8 or 9 rats ran faster following nonrewarded than following rewarded trials. As another index, one especially pertinent here, if one compares the last training day of the 60- and 200-trial groups, the 200-trial group ran faster following nonrewarded trials, yet the 200-trial group extinguished more rapidly. Both findings are consistent with the view of expectancy outlined here.

Shifts from partial to consistent reward. Some years ago, Theios (1962) reported that a shift from irregular partial reward to consistent reward had very little effect on resistance to extinction relative to partial reward alone. This finding was taken to be inconsistent with certain theories that might expect a shift from partial reward to consistent reward to reduce resistance to extinction. Subsequently, a variety of other investigators employing irregular partial reward schedules reported similar findings (e.g., Sutherland, Mackintosh, \& Wolfe, 1965). However, some years later, a rather different finding was reported by Campbell, Knouse, and Wroten (1970), who found that a shift from a single alternation schedule to consistent reward increased resistance to extinction relative to single alternation training alone. Since Campbell et al, also reported that single alternation alone produced greater resistance to extinction than consistent reward alone produced, the paradoxical nature of their finding is apparent: A shift from a schedule that produced greater 
resistance to extinction, single alternation, to one that produced lesser resistance to extinction, consistent reward, increased rather than decreased resistance to extinction. I have recently obtained findings similar to those of Campbell et al. by employing very simple two-trial sequences, a rewarded trial followed by a nonrewarded trial, RN, or two rewarded trials is succession, RR (Capaldi, 1993). We found in our laboratory that a shift from $R N$ to RR increased resistance to extinction relative to $\mathrm{RN}$ alone or $\mathrm{RR}$ alone. RR alone and RN alone produced about equal resistance to extinction.

It is important to note that the shift from single alternation to consistent reward in the Campbell et al. study (1970) and the shift from RN to RR in our studies occurred after the rats had come to strongly anticipate nonreward. In short, at the time of shift in these studies, the retrospective memory $S^{R}$ gave rise to $E^{N}$ or $S^{R} \rightarrow E^{N}$. Perhaps it can be seen why the present expectancy interpretation would expect a shift from single alternation to consistent reward and from RN to RR to elevate resistance to extinction. Very simply, in the shift phase, $S^{\mathbf{R}}$ would elicit $\mathrm{E}^{\mathrm{N}}$, and $\mathrm{E}^{\mathrm{N}}$ would occur on rewarded trials and so would be conditioned to the instrumental response. Thus, when $\mathrm{E}^{\mathrm{N}}$ subsequently occurred in extinction it would tend to elicit responding. Note that $\mathrm{E}^{\mathrm{N}}$ does not become conditioned to the instrumental response under either the consistent reward schedule or the RR schedule given alone. And under either the single alternation schedule or the RN schedule given alone, $\mathrm{E}^{\mathrm{N}}$ is inhibitory, not excitatory.

It is perhaps apparent why a shift from an irregular partial reward schedule to a consistent reward schedule does not increase resistance to extinction. Under an irregular partial reward schedule, transitions from rewarded trials to rewarded trials occur. These RR transitions would have two effects. One is that $\mathrm{E}^{\mathrm{N}}$ would not be as strong in the presence of $S^{\mathbf{R}}$ under partial reward training as under single alternation or RN training. A second is that, to some extent, $E^{N}$ would already have become a signal for reward in the acquisition phase. Thus, on a shift from an irregular partial reward schedule to a consistent reward schedule, $\mathrm{E}^{\mathrm{N}}$ would not become a substantially better signal for reward in the shift phase.

The single alternation to consistent reward and RN to RR shift findings indicate, according to the present hypothesis, that if an $S-$ cue, in the present case $S^{R}$, is rewarded, $\mathrm{E}^{\mathrm{N}}$ will become a signal for reward, thereby elevating resistance to extinction. In a highly provocative and most interesting series of experiments, Haggbloom (1988), among other things, rewarded rats in the presence of an exteroceptive $S-$ cue (e.g., a tactile cue). This produced increased resistance to extinction relative to a variety of controls. I interpret Haggbloom's (1988) findings in the same terms as the shifts from single alternation to consistent reward or RN to RR; in the shift phase, an $\mathrm{S}-$ cue gave rise to $\mathrm{E}^{\mathrm{N}}$, which became a signal for reward. In Haggbloom's case, the $S-$ cue was an ex- teroceptive cue. In our investigations and that of Campbell et al. (1970), the $S$ - cue was an interoceptive cue, $S^{R}$.

\section{The Theoretical Basis of Expectancy}

Within the Hullian tradition, Pavlovian conditioning is considered to be the mechanism of expectancy (see, e.g., Amsel, 1958, 1962, 1967, 1992; Spence, 1956, 1960). Briefly, according to the Hullian view, goalbox cues may function as conditioned stimuli or CSs, with food in the goalbox as the unconditioned stimulus or US; eating is the unconditioned response or UR, with anticipatory eating, $r_{\mathrm{g}}$, being the conditioned response, or CR. This CR may come to be elicited by cues similar to the goal cues (e.g., startbox cues). It is assumed that $r_{\mathrm{g}}$ produces stimuli, $\mathrm{s}_{\mathrm{g}}$, which may be conditioned to the instrumental response. The Pavlovian approach to expectancy is in no way incompatible with a sequential approach. In fact, as will be shown in due course, incorporating into the sequential model the idea that $\mathrm{E}^{\mathbf{N}}$ and $\mathrm{E}^{\mathrm{R}}$ arise via Pavlovian conditioning may produce a variety of advantages.

I assume that retrospective memories such as $\mathbf{S}^{\mathbf{R}}$ and $\mathbf{S}^{\mathbf{N}}$ can function as CSs, acquiring the capacity to elicit a representation of the anticipated US exactly as do conventional CSs. In other words, $\mathrm{E}^{\mathbf{N}}$ and $\mathrm{E}^{\mathrm{R}}$ are representations of the anticipated US, and $S^{R}$ and $S^{N}$ are CSs that elicit those representations. Before I cite some advantages of this assumption, let us consider some findings concerned with overshadowing and blocking which suggest that retrospective memories may function in a manner similar to conventional CSs, such as apparatus cues. If two conventional CSs, A and B, are presented in compound, $A B$, the more salient of the two may reduce the capacity of the other to elicit the CR. This, as previously indicated, is called overshadowing. If $A$ is presented prior to the $A B$ compound, the capacity of $B$ to elicit the $C R$ may be reduced. This is called blocking. A number of studies have shown that $S^{\mathbf{R}}$ and $S^{\mathbf{N}}$ may block or overshadow exteroceptive cues and vice versa (e.g., Haddad et al., 1981; Haggbloom, 1981; Haggbloom \& Tillman, 1980). In these respects, then, $S^{R}$ and $S^{N}$ are similar to conventional CSs (see also Neely \& Wagner, 1974).

Consider another possible point of similarity between $S^{\mathbf{R}}$ and $S^{\mathbf{N}}$ and conventional CSs. Although the data from Pavlovian experiments are not as clear as might be desired, it appears that as the percentage of reward (US presentations) decline, so too does the asymptote of responding or the vigor of the CR (see Mackintosh, 1974). Thus, viewing $S^{R}$ and $S^{N}$ as conventional CSs can be used to explain not merely a variety of serial learning acquisition findings obtained with regular schedules, such as single alternation pattern learning, but, as well, the sort of patterning findings reported by McCain et al. (1962). Recall that McCain et al. reported, employing a $50 \%$ irregular schedule of partial reward, that when given sufficient training, rats ran faster following nonrewarded trials (62\% of nonrewarded trials were followed by rewarded) than follow- 
ing rewarded trials ( $34 \%$ followed by rewarded). Viewing retrospective memories as CSs, we may say that with training rats came to run faster in the presence of $S^{N}$ than $S^{R}$ because $S^{\mathbf{N}}$ was associated with the higher reward percentage and thus elicited the stronger expectancy of reward.

A possible significant advantage of clearly identifying retrospective memories such as $\mathrm{S}^{\mathrm{R}}$ and $\mathrm{S}^{\mathrm{N}}$ as CSs having properties in common with conventional CSs suggests itself. It may be easier than otherwise to understand how, when, and why retrospective memories may come to exercise control over instrumental responding. This is because, once the retrospective memories are seen as CSs, the assumption that they are in competition with other CSs for control over behavior is unavoidable. I will provide two examples of what $I$ have in mind.

We saw that the present expectancy view can explain why a shift from either single alternation to consistent reward or from RN to RR increases resistance to extinction, whereas a shift from irregular partial reward to consistent reward does not. It has also been found that a shift from consistent reward to irregular partial reward decreases resistance to extinction substantially, relative to partial reward alone. This is easily understood in terms of blocking (see Haggbloom, 1983). In the consistent reward phase, two different CSs, the apparatus cues and $\mathrm{S}^{\mathrm{R}}$, are equally valid predictors of reward, so both presumably will acquire some tendency to elicit the instrumental response. In the partial reward phase, $S^{\mathrm{N}}$ will occur following nonrewarded trials and on rewarded trials, but its potential to acquire control over the instrumental response will be reduced because another CS, the apparatus cues, has previously acquired some control over the instrumental response. In short, the apparatus cues block $\mathrm{S}^{\mathbf{N}}$ from acquiring control over responding. Thus, shifts from consistent reward to irregular partial reward decrease resistance to extinction, presumably because of blocking.

Consider another example of two CSs competing for control over the instrumental response, one which is speculative but may turn out to have serious implications for a better understanding of discrimination learning. For this example, let reward occur in, say, a black runway, $\mathrm{S}+$, and let nonreward occur in a white runway, S-. Previously it was indicated that available data suggest that, both early and late in training, $S^{R}$ is retrieved on transitions from $\mathrm{S}+$ to $\mathrm{S}+$ and $\mathrm{S}^{\mathrm{N}}$ is retrieved on transitions from $S-$ to $S-$. Moreover, early in training, $S^{R}$ may be retrieved on transitions from $S+$ to $S-$ and $S^{N}$ may be retrieved on transitions from $S-$ to $S+$, but that is not the case later in training. But, we may ask, what is retrieved somewhat later in training on transitions from $S+$ to $S-$ and from $S-$ to $S+$ ? Although this has not been established as yet, it seems reasonable to believe, on the basis of retrieval findings obtained in a recent discrimination task (Capaldi et al., 1991), that $S^{N}$ is retrieved in $S-$ and $S^{R}$ in $S+$. When this occurs, two cues are valid signals for reward $\left(S^{R}, S+\right)$, and two cues are valid signals for nonreward $\left(S^{N}, S-\right)$. The control acquired over the instrumental response by each cue in each compound will depend on the relative salience of the cues. Blocking may be a factor, since $S+$ may have acquired some prior excitatory control, and $\mathrm{S}-$, some prior inhibitory control. On the other hand, blocking may be less a factor than might be thought. It has been shown that if in a blocking investigation the superimposed cue in the compound (e.g., B) is much more salient than the originally conditioned cue, blocking fails and B acquires substantial control over responding (Hall, Mackintosh, Goodall, \& Dal Martello, 1977). This observation is relevant because it has been shown that cues such as $\mathbf{S}^{\mathbf{R}}$ and $\mathbf{S}^{\mathbf{N}}$ may overshadow exteroceptive cues, which indicates that $S^{\mathbf{R}}$ and $S^{\mathbf{N}}$ are reasonably salient cues (see, e.g., Haggbloom, 1981). At present, there are no data available which clearly indicate what the consequences may be of $S^{R}, S+$ and $S^{N}$, $S-$ being valid signals for reward and nonreward, respectively, in the later stages of discrimination training. A possibility being alluded to here, of course, is that $S^{R}$ and $S^{N}$ may exercise considerable control over discriminative responding under a wide variety of, and possibly all, conditions.

\section{CONCLUSIONS}

The Hull-Sheffield hypothesis ascribed the partial reinforcement extinction effect to fading stimulus traces which could carry over from one trial to the next, providing that the interval between trials was short. As we have seen, this general sort of assumption was retained in the Hullian framework, since unconditioned responses are seen as giving rise to unconditioned stimuli which may persist (see, e.g., Amsel, 1992). In any case, by about 1960 many suggested that intertrial explanations of the Hull-Sheffield sort were passé and should be replaced by intratrial explanations (see, e.g., Amsel, 1962; Lawrence \& Festinger, 1962; Lewis, 1960). An intratrial explanation, it was said, ascribed the partial reinforcement extinction effect to "some kind of learning mechanism developing on each trial in acquisition and having its effect, associatively later in extinction" (Amsel, 1962, p. 310). As I understand the intratrial approach, it suggests that the proper unit of theoretical analysis is the individual trial-as defined, say, by a trip down the runway from startbox to goalbox.

Earlier I identified three sorts of chunks-the trial chunk, the series chunk, and the list chunk. Phenomena such as the partial reinforcement extinction effect were seen as examples of series chunks (see Capaldi, 1992). Counting, as investigated in my laboratory, is an example of a series chunk, with the animal learning, for example, that the signal for nonreward is three rewarded events. It may be that many of the phenomena investigated in animal learning are examples of series chunks. Thus, while the trial chunk is a useful unit of analysis for some purposes, it may be that the more useful unit for most purposes is the series chunk. Clearly, series chunks are involved in varied reward situations. They may also be 
involved in all phases of conventional discrimination learning. For example, we saw that in the early trials of brightness differential conditioning, where, say, reward occurs in S+ and nonreward in S-, available evidence strongly suggests that memories stored in one alternative may be retrieved in either alternative and so a series chunk is being utilized. Evidence also suggests that later in training a memory stored in one alternative might not be retrieved in the other alternative. Does this mean that series chunks are no longer involved? Perhaps not. I went on to speculate that somewhat later in training, as the animal comes to sharply discriminate between the two alternatives, it might retrieve $S^{R}$ only in $S+$ and $S^{N}$ only in $S-$, regardless of the sequence of $S+$ and $S-$ trials. In that event, the animal would be employing two more or less independent series chunks, one for S+ and one for $\mathbf{S}-$. This, of course, is an intertrial explanation.

There is a second sense in which our theorizing applied to, say, brightness differential conditioning is not an intratrial theory as defined by Amsel and others. Earlier in this paper, not only was it suggested that later in training the animal anticipated reward in $\mathrm{S}+$ and nonreward in $\mathrm{S}-$, but $\mathrm{E}^{\mathrm{R}}$, it was said, might be elicited by $\mathrm{S}^{\mathrm{R}}$ as well as by $S+$. And $E^{N}$, it was said, might be elicited by $S^{N}$ as well as by $\mathbf{S}-$, depending on the relative salience of these various stimuli. Thus, expectancy itself may be an intertrial phenomenon in conventional discrimination tasks. Whatever the case in discrimination tasks, expectancy was considered to be an intertrial phenomenon in varied reward tasks, as indicated by the interpretation of data such as shown in Figure 1.

The sequential model is not merely an intertrial model; it is an intertrial model with a vengeance, so to speak. Contrast it with the Hull-Sheffield view, one of the few relatively successful and well-known intertrial models. As indicated, the sequential model postulates memory retrieval, not fading traces. It assumes that the retrospective memories of reward events have a variety of properties, not merely sensory ones. It assumes that $S^{R}$ and $S^{N}$ may be retrieved at retention intervals of hours or days, or that they may not be retrieved at retention intervals of seconds. The sequential model assumes that the animal may remember not only the goal event from the prior trial but also goal events from two or more prior trials. It assumes too that the animal may anticipate not only the goal event to occur in the current trial, but the goal events that are to occur in one or more subsequent trials. On each trial, then, the animal's memory system may be said to have a spread, retrospectively back to prior events and prospectively forward to expected events. The spread is not restricted to series, because, as we have seen, rats can form list chunks as well as series chunks. List chunks have been investigated little. Too, retrospective memories may be encoded as rules.

When science is working properly, all theories are replaced eventually by better theories. Whatever the fate of this or that assumption of the sequential model or the entire sequential model itself, two things seem clear to me. First, a considerable number of phenomena have been reported, some of them cited in this paper, which seem to defy explanation in intratrial terms and cry, so to speak, for some sort of intertrial explanation. Second, it does not seem that we have seen the end of such intertrial phenomena. On the contrary, I expect that phenomena of an intertrial character, which are more complex than those currently available, which are complex enough, will continue to be reported. This would constitute a continuation of a trend begun some 40-50 years ago. One might date the beginning of a serious intertrial approach to the partial reinforcement extinction effect generally or to single alternation pattern learning more specifically. Since single alternation pattern learning was first reported by Tyler et al. (1953), it has been shown that rats are capable of even more complicated behaviors under a wide variety of conditions (e.g., long retention intervals). It seems possible, if not probable, that as more and more data are collected, the theoretical need for an intertrial approach will become more and more apparent.

\section{REFERENCES}

Adams, J. (1984). Learning of movement sequences. Psychological Bulletin, 96, 3-28.

AMSEL, A. (1958). The role of frustrative nonreward in noncontinuous reward situations. Psychological Bulletin, 55, 102-119.

AMSE L, A. (1962). Frustrative nonreward in partial reinforcement and discrimination learning. Psychological Review, 69, 306-328.

AMSE L, A. (1967). Partial reinforcement effects on vigor and persistence. In K. W. Spence \& J. T. Spence (Eds.), The psychology of learning and motivation (Vol. 1, pp. 1-65). New York: Academic Press.

AMSEL, A. (1992). Frustration theory: An analysis of dispositional learning and memory. New York: Cambridge University Press.

BaCON, W. E. (1962). Partial-reinforcement extinction effect following different amounts of training. Journal of Comparative \& Physiological Psychology, 55, 998-1003.

BlaCK, R. W, \& SPENCE, K. W. (1965). Effects of intertrial reinforcement on resistance to extinction following extended training. Journal of Experimental Psychology, 70, 559-563.

Bloom, J. M. , \& CAPALDI, E. J. (1961). The behavior of rats in relation to complex patterns of partial reinforcement. Journal of Comparative \& Physiological Psychology, 54, 261-265.

Bouton, M. E. (1991). Context and retrieval in extinction and in other examples of interference in simple associative learning. In L. Dachowski \& C. F. Flaherty (Eds.), Current topics in animal leaming: Brain, emotion, and cognition (pp. 25-53). Hillsdale, NJ: Erlbaum.

Bouton, M. E. (1993). Context, time, and memory retrieval in the interference paradigms of Pavlovian learning. Psychological Bulletin, 114, 80-99.

Bouton, M. E., Rosengard, C., Achenbach, G. G., Peck, C. A., \& Brooks, D. C. (1993). Effects of contextual conditioning and unconditional stimulus presentation on performance in appetitive conditioning. Quarterly Joumal of Experimental Psychology, 46B, 63-95.

Bower, G. (1981). Theories of learning. Englewood Cliffs, NJ: Prentice-Hall.

Bullock, D. H., \& Sмiтh, W. C. (1953). An effect of repeated conditioning-extinction upon operant strength. Journal of Experimental Psychology, 46, 349-352.

Burns, R. A., \& Gordon, W. U. (1988). Some further observations on serial enumeration and categorical flexibility. Animal Learning \& Behavior, 16, 425-428.

Burns, R. A., \& SANDERs, R. E. (1987). Concurrent counting of two and three events in a serial anticipation paradigm. Bulletin of the Psychonomic Society, 25, 479-481. 
Campbell, P. E., Knouse, S. B., \& Wroten, J. D. (1970). Resistance to extinction in the rat following regular and irregular schedules of partial reward. Journal of Comparative \& Physiological Psychology, 72, 210-215.

CAPALDi, E. J. (1957). The effect of different amounts of alternating partial reinforcement on resistance to extinction. American Journal of Psychology, 70, 451-452.

CAPALDI, E. J. (1958). The effects of different amounts of training on the resistance to extinction of different patterns of partially reinforced responses. Journal of Comparative \& Physiological Psychology, 51, 367-371.

CAPALDI, E. J. (1964). Effect of N-length, number of different N-lengths, and number of reinforcements on resistance to extinction. Journal of Experimental Psychology, 68, 230-239.

CAPALDI, E. J. (1966). Partial reinforcement: A hypothesis of sequential effects. Psychological Review, 73, 459-477.

CaPAlDI, E. J. (1967). A sequential hypothesis of instrumental learning. In K. W. Spence \& J. T. Spence (Eds.), Psychology of learning and motivation (Vol. 1, pp. 67-156). New York: Academic Press.

CAPALDI, E. J. (1985). Anticipation and remote associations: A configural approach. Joumal of Experimental Psychology: Learning, Memory, \& Cognition, 11, 444-449.

CAPaldi, E. J. (1992). Levels of organized behavior in rats. In W. K. Honig \& G. Fetterman (Eds.), Cognitive aspects of stimulus control (pp. 385-404). Hillsdale, NJ: Erlbaum.

CAPALDI, E. J. (1993, May). The basis of prospective memory in retrospective memory. Invited paper presented at the meeting of the Midwestern Psychological Association, Chicago.

Capaldi, E. J., Alptekin, S., Miller, D. J., \& Barry, K. (1992) The role of instrumental responses in memory retrieval in a T-maze. Quarterly Journal of Experimental Psychology, 45B, 65-76.

CAPAldi, E. J., Berg, R., \& Morris, M. D. (1975). Stimulus control of responding in the early trials of differential conditioning. Learning \& Motivation, 6, 217-229.

Capaldi, E. J., Blitzer, R. D., \& Molina, P. (1979). Serial anticipation pattern learning in two-element and three-element series. Bulletin of the Psychonomic Society, 14, 22-24.

CAPALDI, E. J., \& CAPALDI, E. D. (1970). Magnitude of partial reward, irregular reward schedules and a 24-hour ITI: A test of several hypotheses. Joumal of Comparative \& Physiological Psychology, 72 203-209.

CAPALD, E. J., \& HART, D. (1962). Influence of a small number of partial reinforcement training trials on resistance to extinction. Journal of Experimental Psychology, 64, 166-171.

Capaldi, E. J., HarT, D., \& Stanley, L. R. (1963). Effect of intertrial reinforcement on the aftereffect of nonreinforcement and resistance to extinction. Journal of Experimental Psychology, 65, 70-74.

Capaldi, E. J., Leonard, D. W., \& KsIR, C. (1968). A reexamination of extinction rate in successive acquisitions and extinctions. Joumal of Comparative \& Physiological Psychology, 66, 128-132.

CAPAldi, E. J., \& Levy, K. J. (1972). Stimulus control of punished reactions: Sequence of punishment trials and magnitude of reinforcement trials. Learning \& Motivation, 3, 1-19.

CAPALDI, E. J., \& LYNCH, D. (1968). Magnitude of partial reward and resistance to extinction: Effect of N-R transitions. Journal of Comparative \& Physiological Psychology, 65, 179-181.

CAPAldi, E. J., \& Miller, D. J. (1988a). Counting in rats: Its functional significance and the independent cognitive processes that constitute it. Journal of Experimental Psychology: Animal Behavior Processes, 14, 3-17.

Capaldi, E. J., \& Miller, D. J. (1988b). Number tags applied by rats to reinforcers are general and exert powerful control over responding. Quarterly Journal of Experimental Psychology, 40B, 279-297.

Capaldi, E. J., \& Miller, D. J. (1988c). The rat's simultaneous anticipation of remote events and current events can be sustained by event memories alone. Animal Learning \& Behavior, 16, 1-7.

CAPAldi, E. J., Miller, D. J., \& AlPTEkin, S. (1988). Numerical aspects of nonreinforcement: The same-phase nonreinforcement procedure. Animal Learning \& Behavior, 16, 411-416.

Capaldi, E. J., Miller, D. J., \& Alptekin, S. (1989). A conditional numerical discrimination based on qualitatively different reinforcers. Learning \& Motivation, 20, 48-59.
Capaldi, E. J., Miller, D. J., Alptekin, S., \& Barry, K. (1990). Organized responding in instrumental learning: Chunks and superchunks. Learning \& Motivation, 21, 415-433.

Capaldi, E. J., Miller, D. J., Alptekin, S., Barry, K., \& HagGвLоOм, S. J. (1991). Memory retrieval and discrimination learning. Learning \& Motivation, 22, 439-452.

CAPAldi, E. J., \& Molina, P. (1979). Element discriminability as a determinant of serial-pattern learning. Animal Learning \& Behavior, 7, 318-322.

Capaldi, E. J., Nawrocki, T. M., Miller, D. J., \& Verry, D. R. (1985). An examination into some variables said to affect serial learning. Animal Learning \& Behavior, 13, 129-136.

Capaldi, E. J., Nawrocki, T. M., Miller, D. J., \& Verry, D. R. (1986a). Grouping, chunking, memory, and learning. Quarterly Journal of Experimental Psychology, 38B, 53-80.

Capaldi, E. J., Nawrocki, T. M., Miller, D. J., \& Verry, D. R. (1986b). Time between events as a retrieval cue: Recall and the temporal similarity between the storage and retrieval intervals. Journal of Experimental Psychology: Animal Behavioral Processes, 12, 258-269.

Capaldi, E. J., Nawrocki, T. M., \& Verry, D. R. (1983). The nature of anticipation: An inter- and intraevent process. Animal Learning \& Behavior, 11, 193-198.

CAPALDI, E. J., \& Olivier, W. P. (1967). Effect of intertrial reinforcement following a substantial number of consistently rewarded trials. Journal of Experimental Psychology, 75, 135-138.

Capaldi, E. J., \& SPIVEY, J. E. (1963). Effect of goal box similarity on the aftereffect of nonreinforcement and resistance to extinction. Journal of Experimental Psychology, 66, 461-465.

CAPAldI, E. J., \& SPIVEY, J. E. (1964). Intertrial reinforcement and aftereffects at 24-hour intervals. Psychonomic Science, 1, 181-182.

Capaldi, E. J., \& Stanley, L. R. (1963). Temporal properties of reinforcement aftereffects. Journal of Experimental Psychology, 65, 169-175.

Capaldi, E. J., \& Stanley, L. R. (1965). Percentage of reward vs. N-length in the runway. Psychonomic Science, 3, 263-264.

CAPALDI, E. J., \& VERRY, D. R. (1981). Serial order anticipation learning in rats: Memory for multiple hedonic events and their order. Animal Learning \& Behavior, 9, 441-453.

Capaldi, E. J., Verry, D. R., Nawrocki, T. M., \& Miller, D. J. (1984). Serial learning, interitem associations, phrasing cues, interference, overshadowing, chunking, memory, and extinction. Animal Learning \& Behavior, 12, 7-20.

Capaldi, E. J., \& Wilson, N. B. (1968). Intertrial reinforcement: A test of several hypotheses. Psychonomic Science, 13, 169-170.

CAPAldi, E. J., WynN, W., \& TuRner, L. (1962). Decremental and facilitative effects in the straight-alley runway. Journal of Comparative \& Physiological Psychology, 55, 545-549.

CAPALDI, E. J., \& ZiFF, D. R. (1969). Schedule of partial reward and the negative contrast effect. Journal of Comparative \& Physiological Psychology, 68, 593-596.

Chatlosh, D. L, \& Wasserman, E. A. (1992). Memory and expectancy in delayed discrimination procedures. In $\mathrm{I}$. Gormezano \& E. A. Wasserman (Eds.), Learning and memory: The behavioral and biological substrates (pp. 61-79). Hillsdale, NJ: Erlbaum.

Colwill, R. M., \& Rescorla, R. A. (1986). Associative structures in instrumental learning. In G. H. Bower (Ed.), The psychology of learning and motivation (Vol. 20, pp. 55-104). New York: Academic Press.

Compton, D. M. (1991). Serial learning: A review of the behavioral and physiological research with the rat. Neuroscience \& Biobehavioral Reviews, 15, 363-374.

CROWDER, R. C. (1976). Principles of learning and memory. Hillsdale, NJ: Erlbaum.

DALY, H. B. (1991). Changes in learning about aversive nonreward accounts for ontogeny of paradoxical appetitive reward effects in the rat pup: A mathematical model (DMOD) integrates results. Psychological Bulletin, 109, 325-339.

DALY, H. B., \& DALY, J. T. (1982). A mathematical model of reward and aversive nonreward: Its application in over 30 appetitive learning situations. Journal of Experimental Psychology: General, 111, $441-480$. 
D'Amato, M. R. (1991). Comparative cognition: Processing of serial order and serial pattern. In L. Dachowski \& C. F. Flaherty (Eds.), Current topics in animal learning: Brain, emotion, and cognition (pp. 165-185). Hillsdale, NJ: Eribaum.

Davis, H., \& Memmotr, J. (1982). Counting behavior in animals: A critical evaluation. Psychological Bulletin, 92, 547-571.

DAVIS, H., \& PERUSSE, R. (1988). Numerical competence in animals: Definitional issues, current evidence, and a new research agenda. $B e$ havioral \& Brain Sciences, 11, 561-615.

Dickinson, A. (1980). Contemporary animal learning theory. Cambridge: Cambridge University Press.

Diez-Chamizo, V., Sterio, D., \& Mackintosh, N. J. (1985). Blocking and overshadowing between intra-maze and extra-maze cues: A test of the independence of locale and guidance learning. Quarterly Journal of Experimental Psychology, 37B, 235-253.

FountaIN, S. B. (1990). Rule abstraction, item memory, and chunking in rat serial pattern tracking. Journal of Experimental Psychology: Animal Behavior Processes, 16, 96-105.

Fountain, S. B., \& Hulse, S. H. (1981). Extrapolation of serial stimulus patterns by rats. Animal Learning \& Behavior, 9, 381-384.

Gallistel, C. R. (1990). The organization of learning. Cambridge, MA: MIT Press.

Gelman, R., \& Gallistel, C. R. (1978). The child's understanding of number. Cambridge, MA: Harvard University Press.

Gonzalez, R. C., \& Bitterman, M. E. (1969). Spaced-trials partial reinforcement effect as a function of contrast. Journal of Comparative \& Physiological Psychology, 67, 94-103.

Haddad, N. F., Walkenbach, J., Preston, M., \& Strong, R. (1981). Stimulus control in a simple instrumental task: The role of internal and external stimuli. Learning \& Motivation, 12, 509-520.

HAGGBLOOM, S. J. (1980a). Effects of training level and locus on N-R transitions on resistance to discrimination. Psychological Record, 30 , 419-422.

HAGGBLOOM, S. J. (1980b). Resistance to discrimination and subsequent resistance to extinction as a function of the sequence of partial $\mathrm{S}+$ reward in differential conditioning. Animal Learning \& Behavior, 8 , 441-446.

HAGGBLOOM, S. J. (1980c). Reward sequence and reinforcement level as determinants of $\mathrm{S}-$ behavior in differential conditioning. Animal Learning \& Behavior, 8, 424-428.

HAGGBLOOM, S. J. (1981). Blocking in successive differential conditioning: Prior acquisition of control by internal cues blocks the acquisition of control by brightness. Learning \& Motivation, 12, 485-508

HAGGBLOOM, S. J. (1982). Effects of N-R transitions during partial reinforcement pretraining on resistance to discrimination. Animal Learning \& Behavior, 10, 61-64.

HAGGBLOOM, S. J. (1983). Blocking and partial reinforcement: Effects of N-R transitions early vs. late in training on resistance to extinction. Bulletin of the Psychonomic Society, 21, 153-156.

HAGGBLoOM, S. J. (1985). Serial learning and transfer in rats: Effects of changes in stimulus-stimulus associations, pattern structure, and serial position information. Animal Learning \& Behavior, 13, 370-374.

HAGGBLOOM, S. J. (1988). The signal-generated partial reinforcement extinction effect. Journal of Experimental Psychology: Animal Be havior Processes, 14, 89-95.

Haggbloom, S. J., Birmingham, K. M., \& Scranton, D. L. (1992). Hierarchical organization of series information by rats: Series chunks and list chunks. Learning \& Motivation, 23, 183-199.

HaGGBloom, S. J., \& BRooks, D. M. (1985). Serial anticipation and pattern extrapolation in rats as a function of element discriminability. Animal Learning \& Behavior, 13, 303-308.

HagGbloom, S. J., \& CaPaldi, E. J. (1992). Effects of percentage of reinforcement and number of reinforcements in $\mathrm{S}+$ on discrimination learning in the runway. Bulletin of the Psychonomic Society, $\mathbf{3 0}$, 283-286.

Haggbloom, S. J., \& Tillman, D. J. (1980). Sequential effects on discrimination reversal. Learning \& Motivation, 11, 318-338.

Hall, G., Mackintosh, N. J., Goodall, G., \& Dal Martello, M. (1977). Loss of control by a less valid or by a less salient stimulus compounded with a better predictor of reinforcement. Learning \& Motivation, 8, 145-158.
Hull, C. L. (1930). Knowledge and purpose as habit mechanisms. Psychological Review, 37, 511-525.

HuLl, C. L. (1931). Goal attraction and directing ideas conceived as habit phenomena. Psychological Review, 38, 487-506.

Hull, C. L. (1943). Principles of behavior. New York: AppletonCentury-Crofts.

Hull, C. L. (1952). A behavior system: An introduction to behavior theory concerning the individual organism. New Haven, CT: Yale University Press.

HuLse, S. H. (1980). The case of the missing rule: Memory for reward vs. formal structure in serial-pattern learning by rats. Animal Learning \& Behavior, 8, 689-690.

HULSE, S. H., \& Dorsky, N. P. (1977). Structural complexity as a determinant of serial pattern learning. Learning \& Motivation, 8, 488-506.

Jenkins, W. O., \& Stanley, J. C. (1950). Partial reinforcement: A review and critique. Psychological Bulletin, 47, 193-234.

Jobe, J. B., \& Mellgren, R. L. (1974). Successive nonreinforcements (N-Length) and resistance to extinction at spaced trials. Journal of Experimental Psychology, 103, 652-657.

Jobe, J. B., Mellgren, R. L., Feinberg, R. A., Littlejohn, R. L., \& RIGBY, R. L. (1977). Patterning, partial reinforcement, and N-length as a function of reinstatement of retrieval cues. Learning \& Motivation, 8, 77-97.

LASHLEY, K. S. (1942). An examination of the continuity theory as applied to discriminative learning. Journal of General Psychology, 26, 241-265.

LASHLEY, K. S., \& BALL, J. (1929). Spinal conduction and kinesthetic sensitivity in the maze habit. Journal of Comparative Psychology, 9, 71-105.

Lauer, D. W., \& CARTerette, T. S. (1957). Changes in response measures over repeated acquisitions and extinctions of a running habit. Journal of Comparative \& Physiological Psychology, 50, 334-338.

Lauer, D. W., \& Estes, W. K. (1955). Successive acquisitions and extinctions of a jumping habit in relation to schedule of reinforcement. Journal of Comparative \& Physiological Psychology, 48, 8-13.

LAWRENCE, D. H., \& Festinger, L. (1962). Deterrents and reinforcement. Stanford, CA: Stanford University Press.

LEONARD, D. W. (1969). Amount and sequence of reward in partial and continuous reinforcement. Journal of Comparative \& Physiological Psychology, 67, 204-211.

LeONARD, D. W., \& CAPALDI, E. J. (1971). Successive acquisitions and extinctions in the rat as a function of number of nonrewards in each extinction session. Journal of Comparative \& Physiological Psychology, 74, 102-107

LewIS, D. J. (1960). Partial reinforcement: A selective review of the literature since 1950. Psychological Bulletin, 57, 1-28.

Lobaugh, N. J., Greene, P. L., Grant, M., Nick, T., \& Amsel, A. (1989). Patterned (single) alternation in infant rats after combined or separated lesions of hippocampus and amygdala. Behavioral Neuroscience, 103, 1159-1167.

Mackintosh, N. J. (1974). The psychology of animal learning. Lon don: Academic Press.

Mackintosh, N. J., \& Dickinson, A. (1979). Instrumental (Type II) conditioning. In A. Dickinson \& R. A. Boakes (Eds.), Mechanisms of learning and motivation: A memorial volume to Jerzy Konorski (pp. 143-169). Hillsdale, NJ: Erlbaum.

McCain, G., Lee, P., \& Powell, N. (1962). Extinction as a function of partial reinforcement and overtraining. Journal of Comparative \& Physiological Psychology, 55, 1004-1006.

MUENZINGER, K. F. (1928). Plasticity and mechanization of the problem box habit in guinea pigs. Journal of Comparative Psychology, $8,45-69$.

MunN, N. L. (1950). Handbook of psychological research on the rat. Boston: Houghton Mifflin.

Neely, J. H., \& WAGNeR, A. R. (1974). Attenuation of blocking with shifts in reward: The involvement of schedule-generated contextual cues. Journal of Experimental Psychology, 102, 751-763.

OVERMan, W. H., JR., \& DotY, R. W. (1980). Prolonged visual memory in macaques and man. Neuroscience, $5,1825-1831$.

Perkins, C. C., JR., \& CaCIOPPo, A. J. (1950). The effect of intermit- 
tent reinforcement on the change in extinction rate following successive reconditionings. Journal of Experimental Psychology, 40, 794-801

Rescorla, R. A., \& Wagner, A. R. (1972). A theory of Pavlovian conditioning: Variations in the effectiveness of reinforcement and nonreinforcement. In A. H. Black \& W. F. Prokasy (Eds.), Classical conditioning II: Current research and theory (pp. 64-99). New York: Appleton-Century-Crofts.

RestLe, F. (1957). Discrimination of cues in mazes: A resolution of the "place-vs.-response" question. Psychological Review, 64, 217-228.

RoBERTs, W. A. (1991). Testing optimal foraging theory on the radial maze: The role of learning in patch sampling. Animal Learning \& Behavior, 19, 305-316.

RoITBLAT, H. L. (1982). The meaning of representation in animal memory. Behavioral \& Brain Sciences, 5, 353-371.

Roitblat, H. L., Pologe, B., \& Scopatz, R. A. (1983). The representation of items in serial position. Animal Learning \& Behavior, 11, 489-498.

Rudy, J. W., Homzie, M. J., Cox, R. D., Graeber, R. C., \& Carter, E. N. (1970). Effects of sequential manipulations in the within-subjects partial reinforcement runway experiment with rats. Journal of Comparative \& Physiological Psychology, 72, 105-115.

SHEFFIELD, V. F. (1949). Extinction as a function of partial reinforcement and distribution of practice. Journal of Experimental Psychology, 39, 511-526.

SkINNER, B. F. (1938). The behavior of organisms. New York: Appleton-Century-Crofts.

SPENCE, K. W. (1936). The nature of discrimination learning in animals. Psychological Review, 43, 427-449.

SPENCE, K. W. (1937). The differential response in animals to stimul varying within a single dimension. Psychological Review, 44, 430-444.

SPENCE, K. W. (1956). Behavior theory and conditioning. New Haven, CT: Yale University Press.

SPENCE, K. W. (1960). Behavior theory and learning. Englewood Cliffs, NJ: Prentice-Hall.

Spence, K. W., Platt, J. R., \& Matsumoto, R. (1965). Intertrial reinforcement and the partial reinforcement effect as a function of number of training trials. Psychonomic Science, 3, 205-206.

Sutherland, N. S., \& Mackintosh, N. J. (1971). Mechanisms of animal discrimination learning. New York: Academic Press.

Sutherland, N. S., Mackintosh, N. J., \& Wolfe, J. B. (1965). Extinction as a function of the order of partial and consistent reinforcement. Joumal of Experimental Psychology, 69, 56-59.

Terrace, H. A. (1991). Chunking during serial learning by a pigeon I. Basic evidence. Journal of Experimental Psychology: Animal Behavior Processes, 17, 81-93.
THeios, J. (1962). The partial reinforcement effect sustained through blocks of continuous reinforcement. Journal of Experimental Psychology, 64, 1-6.

Tolman, E. C. (1932). Purposive behavior in animals and men. New York: Century.

Tyler, D. W., Wortz, E. C., \& Bitterman, M. E. (1953). The effect of random and alternating partial reinforcement on resistance to extinction in the rat. American Journal of Psychology, 66, 57-65.

W AGNER, A. R. (1961). Effects of amount and percentage of reinforcement and number of acquisition trials on conditioning and extinction. Journal of Experimental Psychology, 62, 234-242.

WAgner, A. R. (1963). Overtraining and frustration. Psychological Reports, 13, 717-718.

WAGNER, A. R. (1971). Elementary associations. In H. H. Kendler \& J. T. Spence (Eds.), Essays in neobehaviorism: A memorial volume to Kenneth W. Spence (pp. 187-213). New York: Appleton-CenturyCrofts.

Wagner, A. R., \& BRANDON, S. E. (1989). Evolution of a structured connectionist model of Pavlovian conditioning (AESOP). In S. B. Klein \& R. R. Mowrer (Eds.), Contemporary learning theories: Pavlovian conditioning and the status of traditional learning theory (pp. 149-189). Hillsdale, NJ: Erlbaum.

Wasserman, E. A. (1986). Prospection and retrospection as processes of animal short-term memory. In D. F. Kendrick, M. E. Rilling, \& M. R. Denny (Eds.), Theories of animal memory (pp. 53-75). Hillsdale, NJ: Erlbaum.

Williams, B. A. (1989). Partial reinforcement effects on discrimination learning. Animal Learning \& Behavior, 17, 418-432.

Wilson, W., Weiss, E. J., \& AMSEL, A. (1955). Two tests of the Sheffield hypothesis concerning resistance to extinction, partial reinforcement and distribution of practice. Journal of Experimental Psychology, 50, 51-60.

WOODWORTH, R. S. (1938). Experimental psychology. New York: Holt. Wright, A. A. (1992). Testing the cognitive capacities of animals. In I. Gormezano \& E. A. Wasserman (Eds.), Learning and memory: The behavioral and biological substrates (pp. 45-60). Hillsdale, NJ: Erlbaum.

YaZAWA, H., \& Fujita, O. (1984). Reinforcement pattern learning: Do rats remember all prior events? Animal Learning \& Behavior, 12, 383-390.

(Manuscript received August 5, 1993; accepted for publication January 5, 1994.) 